Copyright (C) 2007 IEEE. Reprinted from

Proceedings of the IEEE, 2007; 95 (8):1528-1558

This material is posted here with permission of the IEEE. Such permission of the IEEE does not in any way imply IEEE endorsement of any of the University of Adelaide's products or services. Internal or personal use of this material is permitted. However, permission to reprint/republish this material for advertising or promotional purposes or for creating new collective works for resale or redistribution must be obtained from the IEEE by writing to pubs-permissions@ieee.org.

By choosing to view this document, you agree to all provisions of the copyright laws protecting it. 


\title{
T-Ray Sensing and Imaging
}

\author{
An Australian research group has investigated a number of terahertz application \\ areas including liquid T-ray spectroscopy, bioaffinity sensing, image rendering, \\ tomography, microwire T-ray transmission, and detection through plastic layers.
}

\author{
By Withawat Withayachumnankul, Gretel M. Png, Student Member IEeE, \\ Xiaoxia Yin, Student Member IEeE, Shaghik Atakaramians, Inke Jones, Hungyen Lin, \\ Benjamin Seam Yu Ung, Jegathisvaran Balakrishnan, Brian W.-H. Ng, Member IeeE, \\ Bradley Ferguson, Member IEeE, Samuel P. Mickan, Member IEeE,
} Bernd M. Fischer, and Derek Авbott, Fellow IEEE

\begin{abstract}
T-ray wavelengths are long enough to pass through dry, nonpolar objects opaque at visible wavelengths, but short enough to be manipulated by optical components to form an image. Sensing in this band potentially provides advantages in a number of areas of interest to security and defense such as screening of personnel for hidden objects and the retection of chemical and biological agents. Several private companies are developing smaller, reliable cheaper systems allowing for commercialization and this motivates us to review a number of promising applications within this paper. While there are a number of challenges to be overcome there is little doubt that T-ray technology will play a significant role in the near future for advancement of security, public health, and defense.
\end{abstract}

KEYWORDS | Biosensing; femtosecond laser systems; microwires; near-field phenomena; retection; sub-wavelength effects; terahertz; tomography; T-ray imaging; T-rays; waveguides

\footnotetext{
Manuscript received July 22, 2005; revised April 29, 2007. This program was supported by The Australian Research Council (ARC), the Sir Ross and Sir Keith Smith Fund, the Defence Science and Technology Organisation (DSTO), and NHEW P/L. W. Withayachumnankul, G. M. Png, X. Yin, S. Atakaramians, I. Jones, H. Lin, B. S. Y. Ung, J. Balakrishnan, B. W.-H. Ng, B. M. Fischer, and D. Abbott are with the Centre for Biomedical Engineering (CBME) and the School of Electrical \& Electronic Engineering, The University of Adelaide, Adelaide, SA 5005, Australia (e-mail: withawat@eleceng.adelaide.edu.au; gpng@eleceng.adelaide.edu.au; xxyin@eleceng.adelaide.edu.au; shaghik@eleceng.adelaide.edu.au; ijones@eleceng. adelaide.edu.au; hlin@eleceng.adelaide.edu.au; bung@eleceng.adelaide.edu.au; jega@eleceng.adelaide.edu.au; bwng@eleceng.adelaide.edu.au; bfischer@eleceng. adelaide.edu.au; dabbott@eleceng.adelaide.edu.au).

B. Ferguson is with the Centre for Biomedical Engineering (CBME) and the School of Electrical \& Electronic Engineering, The University of Adelaide, Adelaide, SA 5005 Australia. He is also with Tenix Systems Pty Ltd., Mawson Lakes, SA 5095, Australia (e-mail: brad.ferguson@tenix.com).

S. P. Mickan is with the Centre for Biomedical Engineering (CBME) and the School of Electrical \& Electronic Engineering, The University of Adelaide, Adelaide, SA 5005, Australia. He is also with Davies Collison Cave, Melbourne, VIC 3000, Australia (e-mail: spmickan@eleceng.adelaide.edu.au).

Digital Object Identifier: 10.1109/JPROC.2007.900325
}

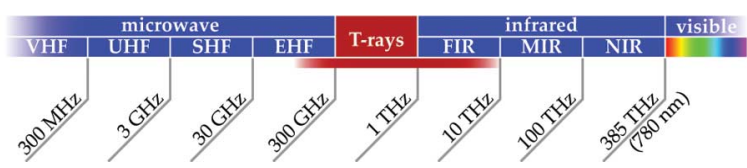

Fig. 1. The T-ray band and its neighboring designations. The T-ray band is loosely defined to be between 0.1 and $10 \mathrm{THz}$, the lower part and upper part of which overlap with the conventional designations of EHF microwave (millimeter wave) and far infrared, respectively.

\section{INTRODUCTION}

The T-ray region of the electromagnetic spectrum has proven to be one of the most elusive. T-ray radiation is loosely defined by the 0.1 to $10 \mathrm{THz}$ frequency range (where $1 \mathrm{THz}$ is $10^{12}$ cycles/s). Situated between infrared (IR) light and millimeter wave radiation (see Fig. 1), the terahertz gap is resistant to the techniques commonly employed in these well-established, neighboring bands. Inefficient generation techniques and high atmospheric absorption constrained early interest and funding for THz gap science [1]-[3].

In the early to mid-1990s, however, the T-ray region became more accessible due to ultrafast pulsed sources and detectors based on pulsed laser excitation, which generate and detect free space T-ray radiation [4]. Two broad classes of T-ray generation and detection relying on ultrafast laser pulses have been developed. The first, using photoconductive antennas (PCAs), was reported by Mourou et al. [5] in the $\mathrm{GHz}$ range and then extended into the $\mathrm{THz}$ region by Auston et al. [6]. This method was further developed at Bell Labs [7] and the IBM Watson Research Center [8], and is now used in a commercial product from Picometrix Inc, MI [9], [10]. The second method, using the nonlinear effects that exploit optical rectification (OR) 
and electrooptic sampling (EOS), was first observed by $\mathrm{Hu}$ et al. [11] and $\mathrm{Wu}$ et al. [12], and used in a commercial product by Zomega Terahertz Corp, NY [13]. Large-scale stand-alone imaging systems became available in 2002 from TeraView Ltd. [14] and Nikon Corp. [15]. As the $\mathrm{THz}$ gap has been opened up, T-rays are being used for an increasing range of sensing and imaging applications.

In general, nonpolar, dry, and nonmetallic materials are transparent or translucent to T-ray radiation. Therefore, weapons concealed beneath clothing or products contained in plastic packages can be seen by T-ray sensors. This transparency motivates the use of T-rays in quality control and security applications [16]-[18]. The strong absorption of T-ray energy by water molecules, which complicates measurements in open-air settings, has merits in biology, where T-rays are highly sensitive to the hydration level in biological tissue [19], [20]. Furthermore, the application of T-rays to human tissue is harmless, as T-rays are nonionizing [21].

Regarding fundamental science, T-rays are of great importance to material spectroscopy. Essentially, most polar molecules either in the solid or liquid phase absorb unique T-ray energies for their intermolecular vibrational transitions [22]-[24], whereas polar molecules in the gas phase have their rotational transition energies spanning the microwave and T-ray frequencies [25]-[27]. The absorption spectrum across this range, therefore, allows material characterization, classification or recognition by means of T-ray spectroscopy [28], [29]. Moreover, moderately doped semiconductors also have plasma frequencies and damping rates in the T-ray frequency region, i.e., between 0.1 and $2.0 \mathrm{THz}$ [30], [31]. The plasma frequency and the damping rate are proportional to the carrier density and mobility of semiconductors, respectively [34] thus, T-ray spectroscopy is ideal for the study of carrier dynamics in semiconductors. T-rays are also used to stimulate Rabi oscillations in two-level impurity states in semiconductors, which enables the manipulation of physical qubits [33]-[35].

This paper aims to provide readers a review of research carried out by the Adelaide T-ray group, with collaborators, during the past few years. Initiated by the researchers from several disciplines, the research covers a wide range of T-ray applications, from biology, medicine, chemistry, material science, and security, to fundamental hardware. As such, the paper is divided into several independent sections as follows:

- Section II briefly discusses the potential sources of uncertainty in widely used terahertz time-domain spectroscopy (THz-TDS) systems. The target material's optical constants at T-ray frequencies, determined from THz-TDS, are affected by these sources of uncertainty. The mathematical relations between the source variances and the optical constant variances are derived.
- Section III gives an overview of the value of different molecular modeling techniques for the prediction of vibrational modes, especially in the mid-infrared and terahertz region. There is a wide range of different levels of theory available for molecular modeling - the choice depending on the kind of system to be investigated. For our calculations we apply different theoretical approaches such as Hartree-Fock and density functional theory using readily available electronic structure programs: Gamess-US and Gaussian03.

- Section IV reviews the methods used for T-ray liquid spectroscopy, including the limitations on the SNR of this technique and proposed improvements.

- Section $V$ presents a new bioaffinity sensor based on double-modulated differential time-domain spectroscopy (DTDS). The sensor is fabricated from biotin which is able to effectively bind with the target molecules, avidin. The high sensitivity of double-modulated DTDS enables detection of ultrathin bound avidin layers in the order of a few micrometers.

- Section VI introduces existing research into T-ray biotissue inspection and modeling, and features one modeling technique that could be used to predict T-ray behavior in layered media. For T-rays to mature into a trusted medical diagnostic tool, it is paramount to understand T-ray interaction with biotissue. Heterogeneous and layered, biotissue are particularly complex to model.

- Section VII investigates microwire fibers for lowloss terahertz transmission. Microwires, air-clad wire waveguides with diameters smaller than the operating wavelength (a few $\mu \mathrm{m}$ ), have an enhanced evanescent field resulting in a low loss waveguide structure for the terahertz frequency regime. Based on our experimental data for the bulk material absorption of diamond, glass, and polymer, we calculate the normalized field distribution, power fraction outside the wire and effective loss.

- Section VIII provides a comprehensive review on the different T-ray near-field imaging techniques in the literature that can be broadly classified into three groups: aperture, tip, and highly focused beam methods.

- Section IX highlights two possible rendering techniques and the result of applying them to T-ray data. Innovative T-ray data rendering techniques allow analysis of T-ray data to advance beyond conventional time- and frequency-domain plots. A plethora of established rendering and image enhancement techniques exist in the optical frequency range-some of which may be adaptable for T-rays. 
- Section X demonstrates 3-D T-ray tomography techniques. T-ray computed tomography or diffraction tomography yields cross-sectional images of dielectric targets based on transmission mode T-ray functional imaging. An ensemble of the images is used to determine the 3-D shape of the object, visualized by a surface rendering technique.

- Section XI, as an extension to Section X, approaches T-ray local tomography with a waveletbased reconstruction algorithm. This algorithm can be used to recover the area of interest for rapid T-ray measurement, compared with a traditional reconstruction algorithm. An image recovered from approximate and detailed reconstruction coefficients using the current algorithm is also illustrated.

- Section XII demonstrates the use of T-rays in substance retection for parcels and packages. We outline a case study demonstrating retection of lactose through various plastic layers at room temperature.

\section{THz-TDS MEASUREMENT UNCERTAINTY ANALYSIS}

An ultrafast-pulsed terahertz time-domain spectroscopy (THz-TDS) system is composed of several mechanical, optical, and electronic parts [36], [37], each of which has a limit in precision and a range of uncertainty. In operation, the uncertainty of these parts contributes to the total uncertainty appearing at the output, i.e., the uncertainty in the extracted optical constants of a measured sample.

In this section, we show how the sources of uncertainty propagate to the output, and we derive a mathematical relation between each source variance and the output variance. In addition to the uncertainty arising from the hardware, the analysis also considers sources of uncertainty that might take place throughout the parameter extraction process (for more information about the parameter extraction process, see, e.g., [38], [39]).

The assumptions of the THz-TDS measurement considered here are that: i) a sample under measurement is a dielectric slab with parallel, flat surfaces; ii) scattering and reflections at the surfaces are negligible; iii) the incident angle of the T-ray beam is normal to the sample surface; and iv) the reference signal is measured under the same conditions, except for the absence of the sample.

Fig. 2 shows propagation of uncertainties during the THz-TDS measurement and the parameter extraction process. The first uncertainty involves error in positioning of the delay line stage, which delays the optical probe pulse by using a pair of moving mirrors. This uncertainty affects the sampling time of an optically gated detector. Combined with electronic and optical noise, the uncertainty in the sampling time gives rise to uncertainty in the sampled T-ray pulse amplitude.

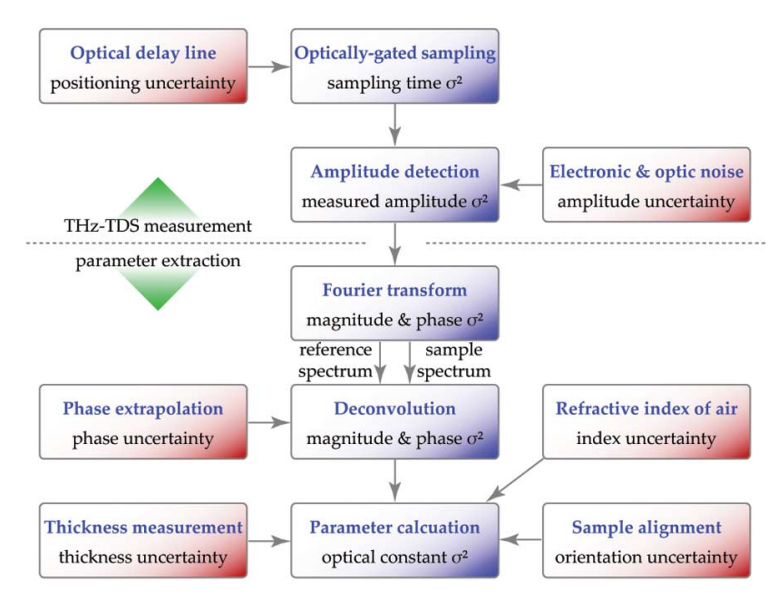

Fig. 2. Propagation of uncertainties. The uncertainty sources (red boxes) can take place in both the THz-TDS measurement and the parameter extraction process. They eventually contribute to the variance in the extracted optical constants.

When measured T-ray signals, including reference and sample signals, undergo signal processing to extract the optical constants, the amplitude error propagates through the Fourier transform and deconvolution stages. An additional uncertainty occurs during the process of phase unwrapping.

The parameter extraction process requires knowledge of the sample's thickness and its orientation. This step introduces uncertainty in the thickness measurement and uncertainty in the sample alignment. In addition, the error in air refractive index estimation contributes to the overall uncertainty. Eventually, all these errors accumulate and contribute to the uncertainty in the measured optical constants.

Before the mathematical expressions of uncertainty are given, the notation used is summarized in Table 1 . The analytical expression for each individual source of uncertainty that contributes to optical constant variance is as follows:

1) Amplitude Uncertainty: Given the amplitude variance of time-domain reference and sample signals, denoted by $\sigma_{E_{\text {ref }}}^{2}(k)$ and $\sigma_{E_{\text {sam }}}^{2}(k)$, respectively, the amplitude-related variances in the optical constants read

$$
\begin{array}{r}
\sigma_{n, E}^{2}(\omega)=\left[\frac{c}{\omega d}\right]^{2}\left\{\frac{A(\omega)}{\left|E_{\mathrm{sam}}(\omega)\right|^{4}}+\frac{B(\omega)}{\left|E_{\mathrm{ref}}(\omega)\right|^{4}}\right\}, \\
\sigma_{\alpha, E}^{2}(\omega)=\frac{4}{d^{2}}\left\{\frac{C(\omega)}{\left|E_{\mathrm{sam}}(\omega)\right|^{4}}+\frac{D(\omega)}{\left|E_{\mathrm{ref}}(\omega)\right|^{4}}\right. \\
\left.+\left(\frac{n(\omega)-n_{0}}{n(\omega)+n_{0}}\right)^{2} \frac{\sigma_{n, E}^{2}(\omega)}{n(\omega)^{2}}\right\},
\end{array}
$$


Table 1 Notation for uncertainty Analysis

\begin{tabular}{cl}
\hline Symbol & Description \\
\hline$c$ & speed of light in vacuum \\
$j$ & $\sqrt{-1}$ \\
$\Re^{2}$ & square of real part \\
$\Im^{2}$ & square of imaginary part \\
$\omega$ & angular frequency \\
$n_{0}$ & index of refraction of air \\
$n(\omega)$ & index of refraction of material \\
$\kappa(\omega)$ & extinction coefficient of material \\
$\alpha(\omega)$ & absorption coefficient of material, $\alpha=2 \omega \kappa / c$ \\
$l$ & thickness of material \\
$\theta_{t}$ & tilted angle of the sample (see Fig. 3) \\
$d$ & path length in material, $d=l / \cos \theta_{t}$ \\
$k$ & time index, running from 0 to $N-1$ \\
$\tau$ & sampling interval \\
$E_{\mathrm{ref}}(\omega)$ & reference signal's complex amplitude \\
$E_{\text {sam }}(\omega)$ & sample signal's complex amplitude \\
$\phi_{c}(\omega)$ & $\angle E_{\text {sam }}(\omega)-\angle E_{\text {ref }}(\omega)$ \\
$\sigma_{n}^{2}(\omega)$ & variance in index of refraction \\
$\sigma_{\alpha}^{2}(\omega)$ & variance in absorption coefficient \\
\hline
\end{tabular}

where

$$
\begin{aligned}
& A(\omega)=\sum_{k=0}^{N-1} \Im^{2}\left[E_{\mathrm{sam}}(\omega) \exp (j \omega k \tau)\right] \sigma_{E_{\mathrm{sam}}}^{2}(k), \\
& B(\omega)=\sum_{k=0}^{N-1} \Im^{2}\left[E_{\mathrm{ref}}(\omega) \exp (j \omega k \tau)\right] \sigma_{E_{\mathrm{ref}}}^{2}(k), \\
& C(\omega)=\sum_{k=0}^{N-1} \Re^{2}\left[E_{\mathrm{sam}}(\omega) \exp (j \omega k \tau)\right] \sigma_{E_{\mathrm{sam}}}^{2}(k), \\
& D(\omega)=\sum_{k=0}^{N-1} \Re^{2}\left[E_{\mathrm{ref}}(\omega) \exp (j \omega k \tau)\right] \sigma_{E_{\mathrm{ref}}}^{2}(k) .
\end{aligned}
$$

The summation is carried out over the time duration of a recorded T-ray signal. In the equations, the amplitude variance $\sigma_{E}^{2}(k)$ incorporates laser-instability fluctuations, delay-line positioning error, thermal noise, background noise, etc. Although the errors from different sources evaluated here are combined, each error can be considered independently.

2) Sample Thickness Uncertainty: Given the sample thickness variance $\sigma_{l}^{2}$, the thickness-related variances of the optical constants are

$$
\begin{aligned}
\sigma_{n, l}^{2}(\omega)= & {\left[\frac{c}{\omega^{2}} \phi_{c}(\omega) \cos \theta_{t}\right]^{2} \sigma_{l}^{2} } \\
\sigma_{\alpha, l}^{2}(\omega)= & {\left[\frac{\alpha(\omega)}{l}\right]^{2} \sigma_{l}^{2} } \\
& +\left[\frac{2}{n(\omega) d}\left(\frac{n(\omega)-n_{0}}{n(\omega)+n_{0}}\right)\right]^{2} \sigma_{n, l}^{2}(\omega) .
\end{aligned}
$$

3) Sample Alignment Uncertainty: This scenario is illustrated in Fig. 3. Given the variance of the sample alignment $\sigma_{\theta_{\mathrm{t}}}^{2}$, the adjustment-related variances of the optical constants are

$$
\begin{aligned}
\sigma_{n, \theta}^{2}(\omega)= & {\left[\frac{c}{\omega d} \phi_{c}(\omega) \tan \theta_{t}\right]^{2} \sigma_{\theta_{t}}^{2} } \\
\sigma_{\alpha, \theta}^{2}(\omega)= & {\left[\alpha(\omega) \tan \theta_{t}\right]^{2} \sigma_{\theta_{t}}^{2} } \\
& +\left[\frac{2}{n(\omega) d}\left(\frac{n(\omega)-n_{0}}{n(\omega)+n_{0}}\right)\right]^{2} \sigma_{n, \theta}^{2}(\omega)
\end{aligned}
$$

4) Air Refractive Index Uncertainty: Given the variance in the air refractive index $\sigma_{n_{0}}^{2}$, the air index-related variances of the optical constants are

$$
\begin{aligned}
\sigma_{n, n_{0}}^{2}(\omega) & =\sigma_{n_{0}}^{2} \\
\sigma_{\alpha, n_{0}}^{2}(\omega) & =\left[\frac{2}{d}\left(\frac{n(\omega)-n_{0}}{n(\omega)+n_{0}}\right)\right]^{2} \times\left[\frac{1}{n(\omega)^{2}}+\frac{1}{n_{0}^{2}}\right] \sigma_{n_{0}}^{2} .
\end{aligned}
$$

The total variance in refractive index and absorption coefficient is given by

$$
\begin{aligned}
& \sigma_{n}^{2}(\omega)=\sigma_{n, E}^{2}(\omega)+\sigma_{n, l}^{2}(\omega)+\sigma_{n, \theta}^{2}(\omega)+\sigma_{n_{0}}^{2}(\omega), \\
& \sigma_{\alpha}^{2}(\omega)=\sigma_{\alpha, E}^{2}(\omega)+\sigma_{\alpha, l}^{2}(\omega)+\sigma_{\alpha, \theta}^{2}(\omega)+\sigma_{\alpha, n_{0}}^{2}(\omega)
\end{aligned}
$$

respectively-where simple addition in quadrature is carried out as the sources of uncertainty are uncorrelated. For further details see [40].

The uncertainty analysis presented in this section enables future investigation of dominant sources of

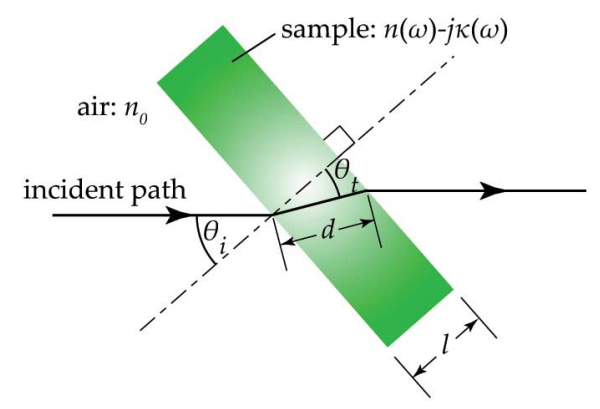

Fig. 3. Tilted sample in a T-ray beam path. The T-ray path inside the sample $d$ is longer than the sample thickness $I$. This exaggerated figure illustrates a small deviation from the normal, which might occur due to sample alignment uncertainty. 
uncertainty in the system, and also enables system optimization. Moreover, a parametric sensitivity analysis can be performed with these equations.

It should, however, be noted that the model is a firstorder approximation, which is valid in the case where sources of uncertainty have variation limited to a small vicinity. In the case of sample alignment uncertainty, the first-order approximation of variance does not result in workable relations for describing the variance of the optical constants. A higher order analysis of uncertainty is thus in progress.

\section{PREDICTION OF THz SPECTRA}

\section{A. Introduction}

Computational chemistry has long been a versatile tool for the prediction of infrared spectra of biomolecules [43]-[48]. However, the assignment of distinct vibrational modes using those calculations remains difficult in the far-infrared and T-ray regions, mainly because the agreement between theoretical and experimental data is often not very consistent and strongly dependent on the molecule. Various studies have shown good agreement between calculated and experimental data [43], [44], [49]-[51]. Recent studies indicate a reliable prediction of the lowest modes in the very far-IR region is not possible with common $a b$ initio molecular modeling techniques or density functional theory (DFT) [52]-[57]. So why is there such an inconsistency of outcomes? In a previous study [58] we investigated the use of ab initio molecular orbital calculations of frequencies in the far-infrared region for the identification of different isomers of the vitamin A derivative, retinal. To further investigate this problem, we performed mid- and far-IR measurements as well as density functional calculations on another molecule also derived from vitamin A, and therefore of similar structure to retinal. We also repeated the retinal calculations using DFT.

There has long been an interest in the low-frequency vibrational modes of these so-called retinoids, especially in all-trans retinal [43], [44], [59], [60]. Retinoids are a group of molecules chemically derived from the dietary supplement vitamin $\mathrm{A}$, formed through oxidation reactions. Retinal or retinaldehyde is fundamental in the transduction of light into visual signals in the photoreceptor level of the retina [61]-[64]. Retinoic acid is a carotenoid organic compound that is a component of visual pigments. It causes cell differentiation, and plays a part in the growth and development of embryos [65]. Medicinally, it is used in tretinoin creams for the treatment of acne and other skin disorders. It is also a component of many commercial products that are advertised as being able to slow skin aging or remove wrinkles [66], [67]. In addition, it is used to treat at least one form of leukemia by causing the blood cells to differentiate, mature, and die [68].

\section{B. Experiments and Calculations}

The mid-infrared measurements $\left(400-1900 \mathrm{~cm}^{-1}\right.$ or 12-57 THz) were performed by a Perkin Elmer BX-1 Fourier-transform infrared spectrometer, which was used with a $1 \mathrm{~mW}$ helium-neon unit and both of the retinoid powders were pressed into $\mathrm{KBr}$ discs. All experiments were carried out at room temperature. Our measurements in the far IR region were performed on a Bruker IFS120HR using transmission through a vaseline mull of the sample supported by polythene plates. Sources employed were either a standard globar ( $>100 \mathrm{~cm}^{-1}$ runs) or a mercury arc source $\left(<100 \mathrm{~cm}^{-1}\right)$. The liquid helium cooled bolometer detector was an Infrared Labs Inc HD3 dewar with a composite silicon bolometer element, also fitted with longpass cold filters at $660 \mathrm{~cm}^{-1}$ or $100 \mathrm{~cm}^{-1}$. Sample preparation was carried out under reduced lighting and the sample mull concentration was kept as high as possible to promote measurable absorbance, while still allowing transmission of light.

For our calculations we used two different electronic structure programs-GAMESS-US [41] and Gaussian03 [42] - to optimize the geometries of two different molecules and to calculate their vibrational frequencies. Both molecules were built in GaussView [69] and the same input matrices were used for the calculations in these electronic structure programs. With both programs, we performed calculations using a hybrid combination of density functional theory (DFT) and HF-B3LYP (Becke's three-parameter hybrid [70] in combination with gradientcorrected correlation functionals by Lee, Yang, and Parr [71]) (6-31G), which is most commonly used.

As can be seen in Figs. 4(a) and 5(a), the calculated vibrational modes in the "fingerprint" (1000-1500 $\mathrm{cm}^{-1}$ or 30-45 THz) region of the spectra of all-trans retinal and all-trans retinoic acid agree well with the experimental data. ${ }^{1}$ Overall, both electronic structure programs produce similar results; furthermore, GAMESS-US appears to be a little more accurate. Looking at the lower frequencies, Figs. 4(b) and 5(b) show the spectra of retinal and retinoic acid below $1000 \mathrm{~cm}^{-1}$. Here, too, good agreement can be seen between calculated and experimental data for alltrans retinal, whereas there seems to be some disagreement below $500 \mathrm{~cm}^{-1}$ for all-trans retinoic acid. Fig. 4(c) focuses on the far-IR region $\left(<200 \mathrm{~cm}^{-1}\right)$ of retinal and shows good agreement between calculated and measured vibrational modes, particularly down to $150 \mathrm{~cm}^{-1}$. Below $150 \mathrm{~cm}^{-1}$ there only seems to be slight agreement (at $41 \mathrm{~cm}^{-1}, 59 \mathrm{~cm}^{-1}$ and $80 \mathrm{~cm}^{-1}$ ), which had already been shown in previous studies [43], [44], [58]. On the other hand, Fig. 5(c) does not show much agreement between experiment and calculation for all-trans retinoic acid in the far-IR region below $400 \mathrm{~cm}^{-1}$.

\footnotetext{
${ }^{1} \mathrm{Ab}$ initio harmonic vibrational frequencies are typically larger than the fundamentals observed experimentally [72].
} 


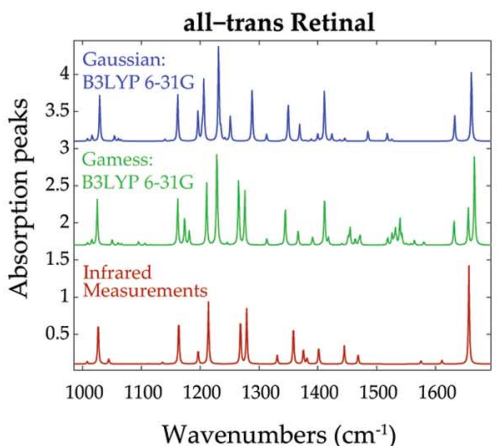

(a)

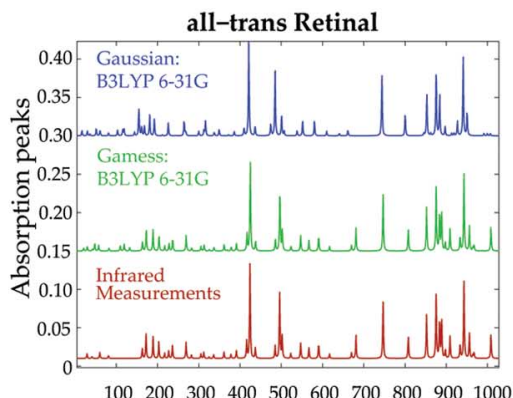

Wavenumbers $\left(\mathrm{cm}^{-1}\right)$

(b)

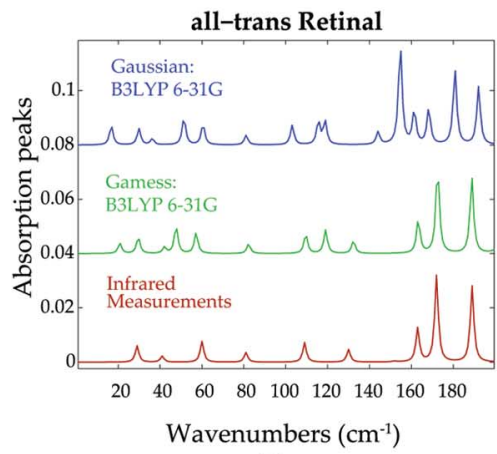

(c)

Fig. 4. Calculated vibrational spectra of all-trans retinal in comparison to our own experimental infrared data covering the mid-IR region of: (a) 1000-1500 $\mathrm{cm}^{-1}$, (b) $<1000 \mathrm{~cm}^{-1}$, and (c) $<200 \mathrm{~cm}^{-1}$. We used two electronic structure packages (Gamess-US [41] and GaussianO3 [42]) and the DFT hybrid combination B3LY.

\section{Discussion}

The above-mentioned calculations of mid- and far-IR vibrational modes use common $a b$ initio molecular orbital theory and density functional theory for two molecules, whose structures only differ in terms of one being an aldehyde and the other an acid. These models predict the far-IR vibrational modes of the aldehyde (retinal), while the same is not possible for the acid (retinoic acid). As possible reasons for this, we hypothesize that all-trans retinoic acid has a stronger tendency to form crystalline structure than all-trans retinal, therefore producing more intermolecular vibrations in the far-infrared region. In these calculations only one isolated molecule is modeled, hence there are no intermolecular vibrations, especially crystal lattice modes, which are common in the far-IR region [29]. Also, these intermolecular vibrations can suppress intramolecular vibrations, which would explain the fact that our calculations produced more peaks than the experiments, both for all-trans retinal and retinoic acid. In general, most basic molecular modeling techniques do not take anharmonicity into account, which has been shown to play an important role in molecular vibrations, especially in the far-infrared region [73] and can therefore be a liability in the prediction of far-IR vibrational spectra.

\section{T-RAY LIQUID SPECTROSCOPY}

Liquid water absorption is one of the most enduring problems facing T-ray imaging and spectroscopy in biomedical applications. The absorption coefficient for liquid water shows a very high T-ray absorption, $200 \mathrm{~cm}^{-1}$ at $1 \mathrm{THz}$ [74]. Over the last decade, there has been a pronounced interest in T-ray spectroscopy of liquids. Liquid spectroscopy allows analysis of chemical composition and provides a better understanding of the solvation dynamics of various types of liquids [75]-[80]. This leads to possible applications such as blood sample screening,

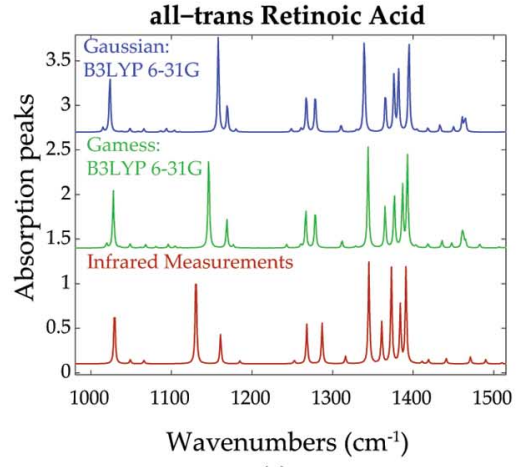

(a)

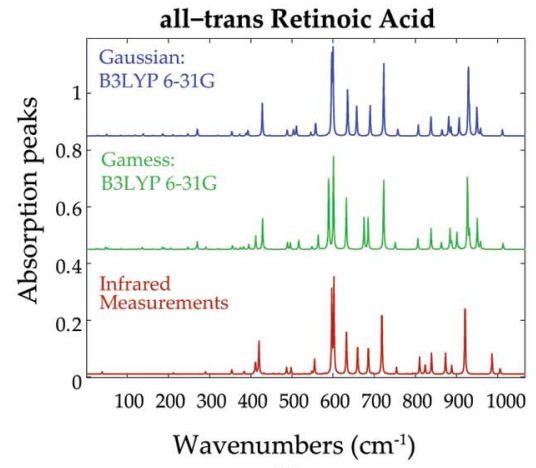

(b)

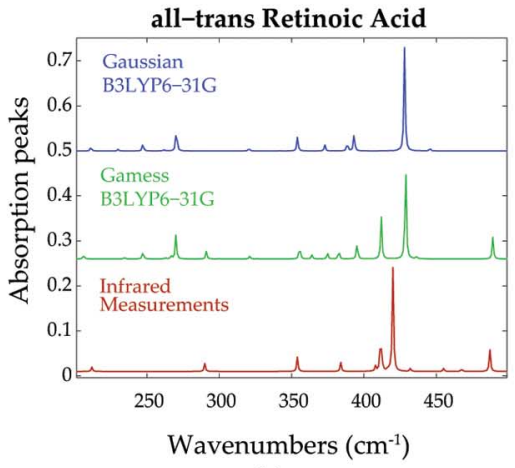

(c)

Fig. 5. Calculated vibrational spectra of all-trans retinoic acid in comparison to our own experimental infrared data covering the mid-IR region of: (a) $1000-1500 \mathrm{~cm}^{-1}$, (b) $<1000 \mathrm{~cm}^{-1}$, and (c) $200-500 \mathrm{~cm}^{-1}$. 
urine testing, contaminant detection in liquids, wet protein sample analysis, detection of inflammable liquids, sugar and salt level of content in water, fat or oil content in polar liquids, and many more applications. Although it has been shown that liquid spectroscopy using T-rays is feasible, there are still open questions that need to be addressed to improve the signal-to-noise ratio (SNR). This section reviews the methods used for T-ray liquid spectroscopy. This section also covers the limitations that affect the SNR of liquid spectroscopy.

\section{A. Liquid Spectroscopy Using THz-TDS}

Terahertz time-domain spectroscopy (THz-TDS) is a conventional technique used for liquid spectroscopy. This technique measures the optical properties of the liquid by taking a reference and a sample reading. In THz-TDS, measurements can take up to several minutes since a separate scan is required for each reading [81]. Within this delay time corresponding laser fluctuations contribute to measurement error. In addition, the error in the estimated thickness of the liquid introduces uncertainties as well.

\section{B. DTDS Technique}

Since noise strongly limits transmission mode measurements of liquids in conventional THz-TDS, a measurement technique called double-modulated liquid differential time-domain spectroscopy (DTDS) was introduced to address the issue [81]. This aimed to improve the accuracy of the liquid parameter measurements by at least an order of magnitude. Double-modulated liquid DTDS involves dithering samples using a shaker at a specific frequency and produces a differential output. Dualthickness measurement by using a thin (reference) and thick (sample) layer of the same liquid is employed in this technique. By modulating the samples rapidly, (swapping between reference and sample) and measuring the waveforms in quick succession, only one scanning delay is required, which reduces the time window in which the laser fluctuates-thus improving measurement accuracy significantly [74], [81], [82]. Based on this technique, improvement in noise performance is observed; however, the dithering frequency of the shaker is limited [83].

In a prototype system, this technique is implemented by using an audio speaker [81]. A square wave is applied to the speaker for the reference and sample switching. The setup diagram for double-modulated liquid DTDS is as shown in Fig. 6. The lock-in amplifiers in the setup diagram, Fig. 7, are used to obtained the mean $y_{m}$ and the amplitude $y_{a}$ signals. The reference $y_{r}$ and the sample $y_{s}$ signals can be obtained through $y_{m}$ and $y_{a}$. Hence the optical parameters of the measured sample can be obtained. Based on this technique, further improvement in SNR is observed; however, the error caused by the uncertainty in the thickness change between the reference and the sample dominates. This is mainly due to the mechanical instability

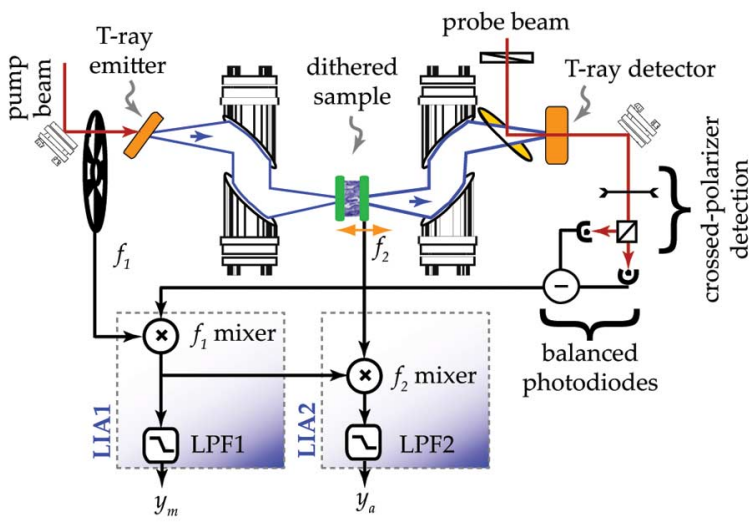

Fig. 6. Setup diagram for liquid spectroscopy using double-modulated DTDS. The dithered sample is placed at the focal point of the T-ray beam and the detected output is a differential signal. This signal is then demodulated using the lock-in amplifiers. Signal processing is required to extract the optical parameters of measured sample. The liquid sample indicated in this figure is inside an HDPE bag and is measured at two thicknesses. The two different thicknesses are achieved by compressive dithering of the bag using an audio speaker. However, the penalty of this technique is that it introduces uncertainty in sample thickness. We therefore propose using exactly the same T-ray set-up, but changing the sample configuration to that of the spinning wheel shown in Fig. 9 that uses two well-defined fixed liquid thicknesses. Here, LPF and LIA indicate a low-pass filter and a lock-in amplifier, respectively. Adapted from [81].

of the audio speaker. Example results of measured optical parameters are shown in Fig. 8.

The plots of extinction coefficient $\kappa$, in Fig. 8, can be obtained by using the following formula:

$$
\kappa=-\ln (\rho) \frac{c_{0}}{\omega d}
$$

where $\rho$ is the magnitude, $\omega$ is the frequency in radians, $c_{0}$ is the speed of light, and $d$ is the thickness change.

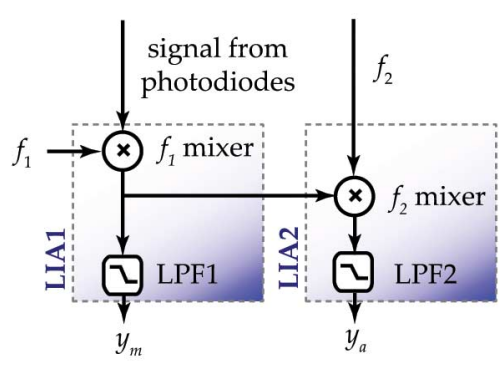

Fig. 7. Block diagram for parameter extraction using two lock-in amplifiers. Based on this setup, the mean $y_{m}$ and the amplitude $y_{a}$ signals can be obtained. The reference $y_{r}$ and the sample $y_{s}$ can be extracted from $y_{m}$ and $y_{a}$. Hence, the optical parameters can be obtained in the usual way. 


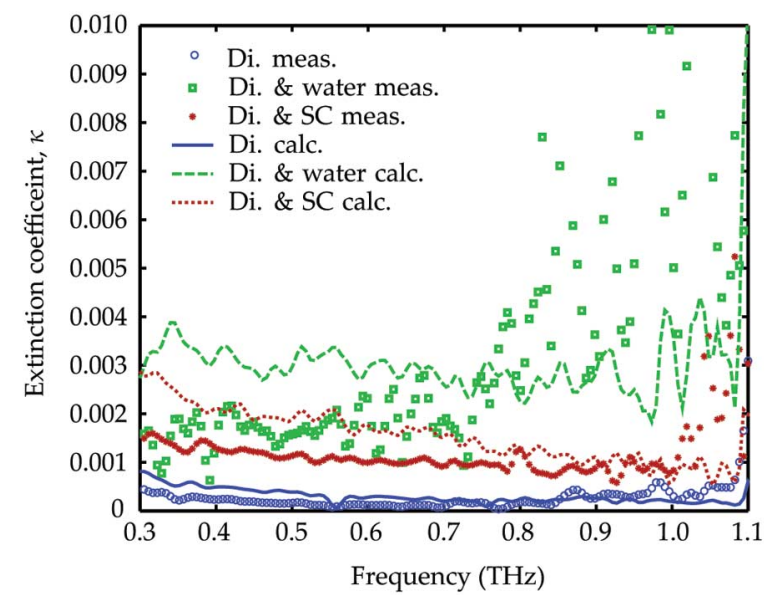

Fig. 8. The graph depicts the extinction coefficient $\kappa$ of different liquid mixtures. This figure compares the calculated (estimated) and measured values of the liquid samples. In this graph "calc" represents theoretical curves and "meas" represents the average of six experimental measurements. The liquid samples are anhydrous dioxane ("Di."), dioxane and water ("Di. \& water"), and dioxane and protein suspension ("Di. \& SC"). Dioxane demonstrated a low absorption and very close to the estimated values. As expected when the protein, Substilisin Carlberg (SC), is mixed with the dioxane, the absorption is observed to increase slightly. As for the mixture of dioxane and water, there is a large gap observed between the measured and the estimated values. This indicates that the SNR of the system is not sufficient to cope with the reduced signal due to water absorption. This motivates us to explore the proposed spinning wheel technique to improve SNR to the level where we can spectroscopically investigate the effects of protein hydration. After [81].

Recently, a method using a spinning wheel has been proposed [84], [85] to address the uncertainty in the thickness change in the dithering technique. The wheel, illustrated in Fig. 9, can be used to determine the characteristics of different types of liquids with an expected improvement in noise performance up to two orders of magnitude. The wheel is designed with a dual thickness geometry, which has fixed thicknesses as compared to the previous dithering technique, and thus reduced noise is expected. The spinning wheel is designed for transmission experiments and therefore requires a window material that has an optimal refractive index, low absorption, and low hygroscopicity.

\section{Window Material}

Different types of window materials have been tested for the spinning wheel, and the results of the refractive index $(n)$ and absorption coefficient $(\alpha)$ at $0.8 \mathrm{THz}$ are shown in Table 2. Two different measurements are carried out on the window materials: i) in a dry condition and ii) in a soaked condition. The motivation of this test is to assist in finding a window material with low hygroscopicity. The window materials are measured via $\mathrm{THz}$ -
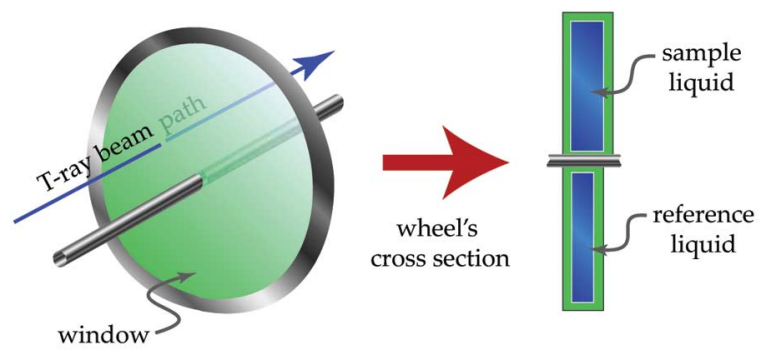

Fig. 9. Spinning wheel with dual-thickness cavity. The idea of the wheel is that it overcomes the uncertainty of the two liquid layer thicknesses produced by the previous audio speaker dithering method. As the wheel has two fixed cavities filled with the sample liquid - one thin and one thick cavity - simple rotation then allows rapid swapping between sample and reference with well-controlled thicknesses. The challenge, however, is to find a suitable window material for the wheel that has both excellent T-ray transmission properties and low hygroscopicity.

TDS before and after soaking in water for at least $48 \mathrm{~h}$. Based on the measurements, the refractive index and the absorption coefficient of the window materials at $0.8 \mathrm{THz}$ are observed. In Table 2, acrylic shows the most significant difference for both the refractive index and the absorption coefficient. Amongst all the materials, cycloolefin copolymer (COC) 8007 shows the most promising result. It has a refractive index of 1.523 before soaking and 1.535 after soaking and an absorption of $0.3 \mathrm{~cm}^{-1}$ before and $0.35 \mathrm{~cm}^{-1}$ after soaking. Fig. 10 illustrates the absorption coefficient of the acrylic and cycloolefin window materials. This figure demonstrates that cycloolefin has very promising optical properties as a window material for liquid spectroscopy. Preliminary results that demonstrate the potential of the spinning wheel technique for liquid spectroscopy using cycloolefin as the window material will be presented in the near future.

\section{T-RAY BIOSENSING}

One of the most successful optical biosensors is currently based on surface plasmon resonance (SPR) [86]. An SPR system monitors the binding of molecules to a sensor by probing refractive index changes of the target layer relying on the principle of bioaffinity, i.e., the affinity between

Table 2 Window Material $n$ and $\alpha\left(\mathrm{cm}^{-1}\right)$ at $0.8 \mathrm{THz}$

\begin{tabular}{|ccccc|}
\hline Material & $n_{\text {dry }}$ & $n_{\text {soaked }}$ & $\alpha_{\text {dry }}$ & $\alpha_{\text {soaked }}$ \\
\hline Acrylic & 1.515 & 1.525 & 7.50 & 9.00 \\
Nylon & 1.534 & 1.537 & 0.25 & 0.50 \\
HDPE & 1.477 & 1.483 & 0.10 & 0.20 \\
Polycarbonate & 1.593 & 1.597 & 5.00 & 6.20 \\
COC 8007 & 1.523 & 1.526 & 0.30 & 0.35 \\
Teflon & 1.443 & 1.447 & 0.10 & 1.50 \\
\hline
\end{tabular}




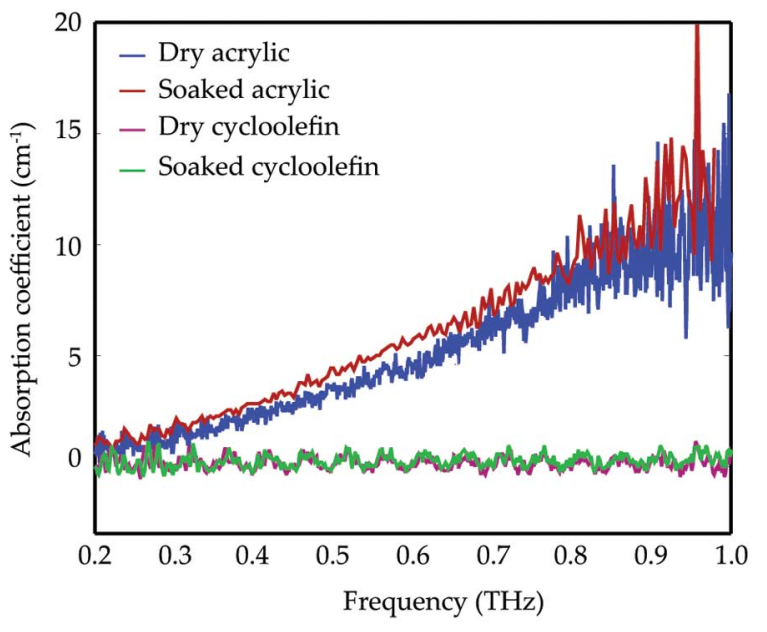

Fig. 10. Absorption of acrylic and cycloolefin (COC8007) window materials before and after a 48 h soak in water. After soaking, excess surface water is removed. The results show that the cycloolefin absorbed negligible water, whereas the acrylic sample demonstrates slight hygroscopicity.

two biomolecules. Bioaffinity can be used in a biosensor by attaching a layer of sensor molecules, or analytes, to a sensor slide. The biosensor slide is used by exposing it to a mixture of unknown molecules, to test if any target molecules, or ligands, are present. SPR detects this new layer by probing the refractive index change with an optical laser beam - a change in refractive index results in a change in the intensity of light reflected off the film at some angle, given by the experiment. SPR relies on the refractive index change at optical wavelengths, as determined by the laser. Certain biomolecules, however, do not show contrast at optical wavelengths, and these molecules could be sensed using T-rays.

A new detection method based on T-rays operating on the same principle as SPR has been proposed, and used successfully to detect minute amounts of label-free DNA molecules [87]. This approach uses integrated T-ray waveguides, incorporating resonant T-ray structures, and has achieved femtomole (fm) sensitivity to label-free DNA hybridization using T-rays [88], [89]. This approach opens up new avenues for label-free detection. However, the integrated waveguides require applicationdependent hardware modifications, delicate sample handling procedures, and the interconnection of a large number of resonators for simultaneous detection. The free space T-ray biosensor proposed in this section would use a standard T-ray spectrometer.

The proposed T-ray biosensor [90] is, like SPR, a bioaffinity detector. Using the highly sensitive technique of double-modulated DTDS [82], [91], a standard T-ray spectrometer is used to detect the binding of target molecules to preprepared sensor molecules, that is, to detect bioaffinity. The molecules used, as ligands (targets) and analytes (sensors) to confirm the technique experimentally, are biotin and avidin.

Biotin is a water-soluble lipid, and is commonly referred to as vitamin $\mathrm{H}-\mathrm{a}$ member of the $\mathrm{B}$-complex group of vitamins. Avidin is a protein that occurs in raw egg white and is remarkable for its high binding affinity for biotin. Avidin and biotin together constitute a pair of biomolecules commonly used in demonstrations of bioaffinity, due to their high binding affinity. Bead-enhanced avidin target molecules, sketched in Fig. 11, create a much thicker molecular layer than avidin target molecules alone. Avidin is enhanced by binding to large, $\mu \mathrm{m}$-sized agarose beads. Agarose is an inert substance, extracted from agar, that is easy to derivitize, or attach to other biomolecules. The agarose beads attached to the avidin amplify the target layer thickness, potentially increasing the biosensor's signal.

The process of sample slide preparation for beadenhanced avidin detection is shown in Fig. 11. The first step in preparing a slide is cleaning the quartz, leaving a surface of hydroxyl $(\mathrm{OH})$ groups. The quartz crystal surface is then modified by self-assembly of the octadecanol molecule, which creates a dense array of hydrophobic strands aligned perpendicularly to the surface. The hydrophobic tails (Fig. 11 in red) of the biotin molecules are then fitted into the hydrophobic octadecanol matrix. The biotin is anchored to the surface and only the charged heads of the biotin molecules are exposed. The detection step is shown in Fig. 11(c), where avidin molecules bind to the biotin layer. In the experiment shown here, the avidin molecules are attached to a large agarose bead, thus the bound target layer has a thickness greater than a layer of bound avidin molecules.

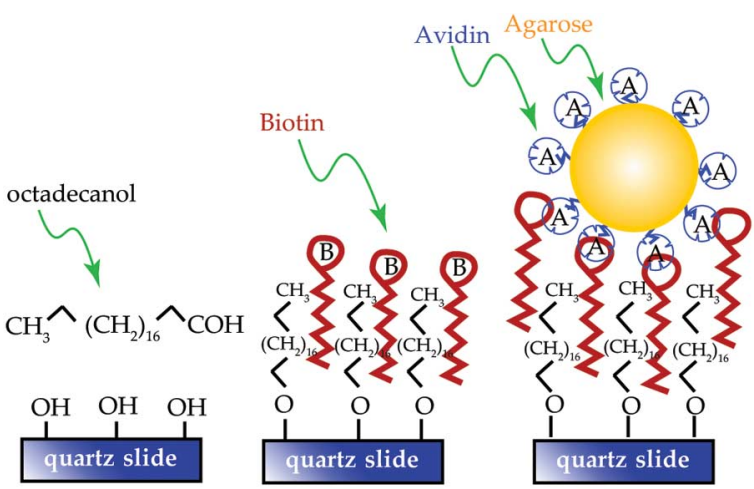

(a)

(b)

(c)

Fig. 11. Biosensor derivitization, preparation, and target detection. This sketch shows the steps involved in the preparation and application of a biotin-avidin bioaffinity slide. (a) Clean sensor slide, and octadecanol. (b) Biotin attached with octadecanol. (c) A vidin on an agarose bead, linking to biotin. After [90]. 
The three time-domain T-ray pulses in Fig. 12 are obtained by measuring the difference signal between (a) biotin and biotin-avidin conjugated agarose, (b) biotin and biotin-avidin complexes without beads, and (c) biotin and biotin-digalactosyldiacyl glycerol : DGDG (control sample). These time-domain pulses are smoothed using a $0.1-1.0 \mathrm{THz}$ band-pass filter. The spectrometer signalto-noise ratio in these measurements is greater than $10^{2}$. The inset shows the waveforms in the Fourier domain (FFT). The intensity of the signal due to inhomogeneities and nonspecific binding is less than the agarose and biotin-avidin signals.

Compared to the similar biotin-avidin experiment without agarose beads, an eightfold enhancement in signal amplitude due to the beads is observed. The signal enhancement is due to the increased refractive index of agarose particles, causing increased T-ray reflection at the biotin-avidin interface. The repeatability of the waveforms, shown in Fig. 12, is tested by measuring the DTDS signal at different lateral positions on each biosensor slide. The signal variation with position is less than $10 \%$.

In this experiment, T-ray DTDS is applied to thin layers of biomolecules for use as a T-ray biosensor. The experimental results indicate that the T-ray spectrometer is highly sensitive to thin layers of target molecules, in the order of submicrometers [90], and can clearly differentiate between target and control samples, even for samples prepared by hand.

\section{MODELING T-RAYS IN LAYERED MEDIA}

T-rays have been applied in medicine to explore possible early or complementary detection techniques of diseases. Ex vivo and in vivo studies of human skin have revealed that

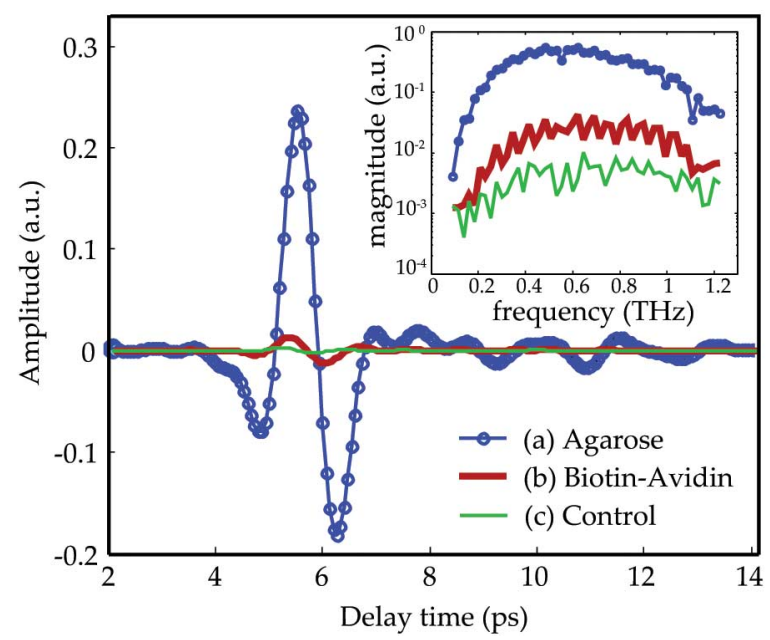

Fig. 12. Measured biosensor waveforms for: (a) agarose or enhanced avidin, (b) biotin detection of avidin, and (c) a control. After [90].
T-rays can detect changes in the health of tissue, such as between excised healthy skin and excised skin with basal cell carcinoma (BCC) [92], and between dry and normal skin in vivo [93]. Excised human and animal tissue have also been inspected with T-rays and their optical properties in the T-ray range show differences between tissue types [94], [95].

Biotissue tends to be stratified and heterogeneous layers are often thinner than the longer T-ray wavelengths, creating a thin-film interference situation. It is impractical to resolve the reflections from every layer, but it is possible to study their collective behavior assuming steady state conditions with no changes to tissue hydration or blood flow. This creates a simplified model with homogeneous layers. Numerical methods have been used to study T-ray propagation in stratified media: the Monte Carlo model and thin-film model [96], finite-difference method [93], and transmission line theory [97], [98]. The mathematics behind transmission line theory assumes a M-layered structure, each layer having its own permittivity $\epsilon_{m}$, conductivity $\sigma_{m}$, permeability $\mu_{m}$, and thickness $h_{m}$. Incident electromagnetic waves can only enter the topmost layer and the outgoing waves can only leave the lowest layer (Mth layer), which has infinite thickness. Reflected waves can therefore only occur on the topmost layer. The boundary condition is that the tangential fields are continuous at each layer interface. The general solution of an incident plane wave in the frequency domain $(\omega)$ can now be solved, and the particular solution is equal to the Fresnel reflection coefficient $R_{\|}$

$$
\begin{aligned}
R_{\|} & =\frac{\text { Amplitude of reflected wave }}{\text { Amplitude of incident wave }} \\
& =\frac{K_{0}-Z_{1}}{K_{0}+Z_{1}}
\end{aligned}
$$

where

$$
\begin{aligned}
Z_{1}= & K_{1} \frac{Z_{2}+K_{1} \tanh \left(u_{1} h_{1}\right)}{K_{1}+Z_{2} \tanh \left(u_{1} h_{1}\right)} \\
Z_{2}= & K_{2} \frac{Z_{3}+K_{2} \tanh \left(u_{2} h_{2}\right)}{K_{2}+Z_{3} \tanh \left(u_{2} h_{2}\right)} \\
& \vdots \\
Z_{m}= & K_{m} \frac{Z_{m+1}+K_{m} \tanh \left(u_{m} h_{m}\right)}{K_{m}+Z_{m+1} \tanh \left(u_{m} h_{m}\right)} \\
& \vdots \\
Z_{M-1}= & K_{M-1} \frac{K_{M}+K_{M-1} \tanh \left(u_{M-1} h_{M-1}\right)}{K_{M-1}+K_{M} \tanh \left(u_{M-1} h_{M-1}\right)} \\
Z_{M}= & K_{M} \operatorname{assuming} h_{M}=\infty \\
K_{m}= & \frac{u_{m}}{\sigma_{m}+j \omega \epsilon_{m}}, \quad \gamma_{m}^{2}=j \omega_{m} \mu_{m} \sigma-\omega^{2} \mu_{m} \epsilon_{m} \\
u_{m}= & \sqrt{\lambda^{2}+\gamma_{m}^{2}}=\sqrt{\lambda^{2}+j \omega_{m} \mu_{m} \sigma-\omega^{2} \mu_{m} \epsilon_{m}} .
\end{aligned}
$$


Table 3 Extrapolated Permittivity and Conductivity at $1.5 \mathrm{THz}$

\begin{tabular}{|ccc|}
\hline & & \\
Layer & $\begin{array}{c}\text { Average relative } \\
\text { permittivity } \epsilon^{\prime}\end{array}$ & $\begin{array}{c}\text { Average } \\
\text { conductivity } \sigma\end{array}$ \\
\hline Skin & 5.6 & 0.39 \\
Adipose Tissue & 20 & 0.9 \\
Bone & 1.5 & 0.1 \\
Dura Mater & 6 & 0.48 \\
CSF & 9.3 & 0.77 \\
Gray Matter & 7.75 & 0.53 \\
White Matter & 6 & 0.4 \\
\hline
\end{tabular}

In the following case study, transmission line theory is used to model one particular stratified part of the body with many distinct layers: the human head. The seven layers modeled were skin, adipose tissue, bone, dura mater, cerebrospinal fluid (CSF), gray matter, and white matter. The optical properties of these tissue layers are mostly available in the microwave frequency region [99], [100] but can be extrapolated into the low T-ray frequency range. Their average relative permittivity $\epsilon^{\prime}$ and average conductivity $\sigma$ in the T-ray frequency range are presented in Table 3. The relative magnetic permeability $\mu$ is assumed to be 1 . The approximate thicknesses of the layers are shown in Fig. 13.

It is acknowledged here that biotissue may introduce scattering. However, a recent study using excised rat tissue has shown that material attenuation due to moisture content is the dominant loss effect in T-ray spectroscopy of biotissue [95]. In addition, this study assumes in vivo T-ray spectroscopy of the head so variations in moisture content over time for excised tissue are not applicable.

In Fig. 14, the reflections correspond to calculated return times from known layer thickness. The inset shows the expected strong reflection from the surface of the skin and the next underlying skin-adipose interface. This waveform agrees with reported experimental results in [93]. In the main plot, the second peak appears to be from the adipose-bone interface. Assuming the average incident T-ray power level is $1 \mathrm{~mW}$ in an amplified T-ray system,

\begin{tabular}{|c|c|}
\hline ir & \\
\hline Skin & f $100 \mu \mathrm{m}$ \\
\hline Adipose tissue & $\uparrow 1000 \mu \mathrm{m}$ \\
\hline Cortical bone & $\downarrow 10,000 \mu \mathrm{m}$ \\
\hline Dura mater & $\downarrow 250 \mu \mathrm{m}$ \\
\hline CSF & $\downarrow 200 \mu \mathrm{m}$ \\
\hline Gray matter & $\uparrow 2000 \mu \mathrm{m}$ \\
\hline White matter & $\downarrow \infty$ \\
\hline
\end{tabular}

Fig. 13. Approximate thickness of seven tissue layers in the head. After Nolte [101].

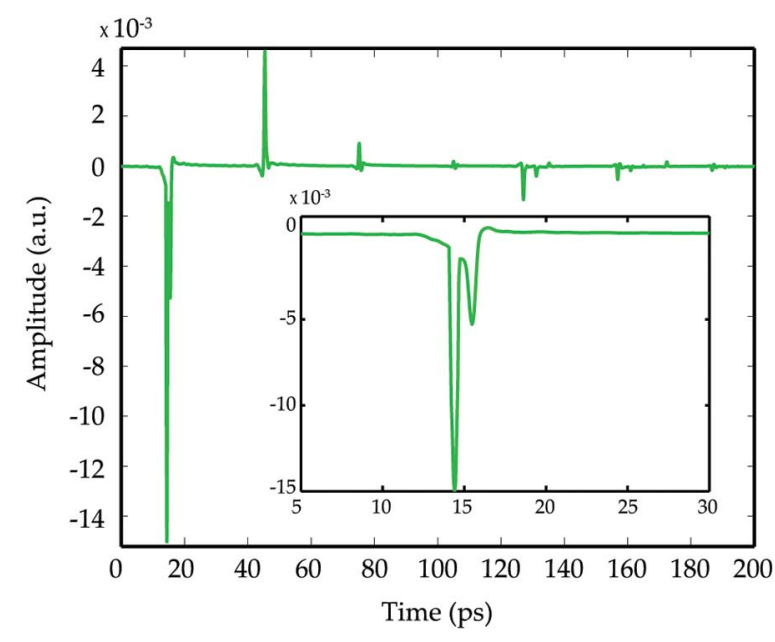

Fig. 14. Simulated time response of the reflected signal from the seven layers of the head. The inset shows a zoomed view from 5 to 30 ps where the first trough is due to the air-skin interface, while the second trough is due to the skin-adipose interface. After [97], [98].

then the second reflection is approximately $8.2 \mu \mathrm{W}$. It is plausible that this second reflection can be detected with present-day technology. The third and subsequent peaks are below $1 \mu \mathrm{W}$, thus they are unlikely to be detected by present T-ray detectors. Higher T-ray power levels, up to $1 \mathrm{~kW}$ [102], may be obtained through the use of synchrotrons and free electron lasers (FELs). The maximum permissible exposure (MPE) limit derived from animal cornea study with T-rays has been reported to be $94 \mathrm{~W}$ (average power per pulse) although for the human body $<20 \mathrm{~W}$ is recommended [103].

Although not all the reflections obtained using the transmission line model are practically detectable, the model is still valid for studying the propagation of T-rays in stratified media and can assist us in developing better detection techniques for future T-ray systems. It would appear that as we approach an input power of $20 \mathrm{~W}$, most of the return pulses, predicted in Fig. 14, are detectable. This is promising for future T-ray scanning of the human head-however, a caveat is that extrapolated parameters have been used in Table 3. Nevertheless, this result provides the motivation for future work to directly measure the parameters in Table 3 within the T-ray regime-and this work is now underway [94], [95].

\section{T-RAY MICROWIRES}

In almost all present-day terahertz time-domain spectroscopy (THz-TDS) systems, free space is used for propagation of terahertz waves. Furthermore, there has been a lack of an alternative to free space as low loss terahertz waveguides have only recently been studied. Several waveguide solutions coming from either electronics or 
photonics have been studied, such as the hollow metallic circular waveguide [104], [105], hollow metallic rectangular waveguide [105], sapphire fiber [106], plastic ribbon waveguide [107], air-filled parallel-plate waveguide [108], [109], plastic photonic crystal fiber [110], coaxial waveguide [111], metal wire waveguide [112], [113], parallelplate photonic waveguide [114], metal sheet waveguide [115], the dielectric-filled parallel-plate waveguide [116], and low-index discontinuity terahertz waveguides (split rectangular and tube waveguides) [117]. In most of these waveguide structures two main mechanisms, i) loss [105] and ii) pulse reshaping and broadening due to dispersion [106], have been reported. However, the main focus of the following analysis in this section will be on the loss mechanism.

To date, metal parallel plates and bare metal wires are promising metal-based guiding techniques reported in the literature with attenuation constants less than $0.3 \mathrm{~cm}^{-1}$ [118] and $0.03 \mathrm{~cm}^{-1}$ [112], respectively. Chen et al. [119] have recently reported loss values less than $0.01 \mathrm{~cm}^{-1}$ near $0.3 \mathrm{THz}$ in plastic fibers. The concept of T-ray guided propagation in these fibers is similar to optical nanowire fibers [120].

Optical nanowires are filaments of dielectric media whose tailorable subwavelength dimensions, in the order of $\mathrm{nm}$, allow a substantial fraction of the guided light (wavelength of $1-1.5 \mu \mathrm{m}$ ) to propagate outside the structure. As a result, in this regime, there exists an enhanced evanescent field outside the nanowire. However, its lowloss optical waveguiding is restricted by sidewall smoothness and diameter uniformity, especially when the diameter of the waveguide is very small [120]. In nanowires, coupling between wires can be achieved via surface contact, with extremely small low-loss waveguide curvature, which is an advantage of using such wires.

Such an enhanced evanescent field behavior occurs for micrometer diameter fibers in the T-ray band, whose wavelength ranges from 30 to $3000 \mu \mathrm{m}$ or equivalently from 0.1 to $10 \mathrm{THz}$. Therefore we coin the term microwires for these waveguides used in the T-ray regime.

\section{A. Bulk Material Measurement}

Here, we present the results of loss and refractive index measurements for a diamond, four glasses (F2, SF6, SF57, and bismuth glass) and a polymer (PMMA) in the terahertz regime and then use these measurements to calculate the normalized electric field distribution, the power fraction outside the fiber, and the effective loss of microwires. To measure the refractive indices and absorption coefficient, we use a commercially available T-ray time-domain spectrometer [121], [122]. The glass samples are well polished on both sides with a cross section of $2 \mathrm{~cm} \times 2 \mathrm{~cm}$ and $0.5 \mathrm{~mm}$ thickness. The diamond crystal is grown under a high pressure high temperature method (manufacturer: Sumitomo) and has a cross section of $3 \mathrm{~mm} \times 3 \mathrm{~mm}$ and $1.5 \mathrm{~mm}$ thickness. The absorption coefficients and refractive indices are obtained by comparing the sample pulses with a reference pulse propagating through dry air. Assuming single mode propagation, the equation for calculating the optical properties can be written in the frequency domain as

$$
\frac{E_{\mathrm{sam}}(\omega)}{E_{\mathrm{ref}}(\omega)}=T_{1} T_{2} C^{2} \exp (-\alpha l / 2) \exp \left(-\mathrm{j} \beta_{0}(n-1) l\right)
$$

where $E_{\text {sam }}(\omega)$ and $E_{\text {ref }}(\omega)$ are the complex components at angular frequency $\omega$ of the sample and reference electric fields, respectively; $T_{1}$ and $T_{2}$ are the total transmission coefficients that take into account the reflections at the entrance and exit faces, respectively; $C$ is the coupling coefficient, the same for the entrance and exit faces; $\beta_{0}$ is the free space phase constant; $\alpha$ is the power absorption coefficient; $n$ the refractive index of the sample; and $l$ is the sample length.

The measured absorption coefficient and refractive indices are shown in Fig. 15(a) and Fig. 15(b), respectively. The bulk material loss caused by the SF6, SF57, and bismuth glasses are close to each other and are higher than that of F2. Note that PMMA and diamond have the lowest material absorption. Individually, the refractive index of each glass sample is significantly higher than the refractive index of the PMMA and diamond samples. This results in a tighter confinement of the T-ray radiation in a glass waveguide because of the increased air-glass index change.

It is obvious that if PMMA is used to make conventional waveguides, it will have lower loss in comparison with the glasses considered here. The question is: what effect do these optical material parameters have on the scaled version of nanowires, i.e., microwires, in the terahertz frequency range?

\section{B. Microwires}

To study this, we use the measured optical parameters to calculate and discuss the normalized electric field distribution, the power fraction outside the fiber, and the effective loss of microwires made up of these materials.

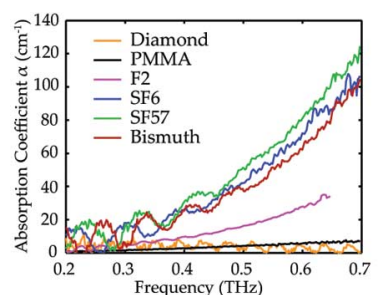

(a)

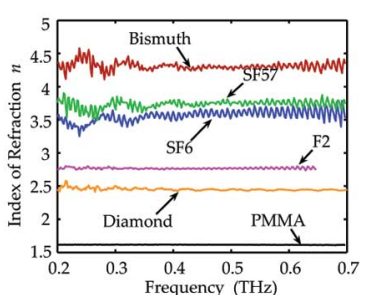

(b)
Fig. 15. (a) Absorption coefficients and (b) the refractive indices of the bulk materials (diamond, PMMA, F2, SF6, SF57, and bismuth glass) measured with a T-ray time domain spectrometer. Adapted from [123]. 
We assume that the wire has a circular cross section, an infinite air cladding, and a step-index profile. It is also assumed that the wire is uniform in diameter and has a smooth sidewall. Considering that the refractive index of air is one and solving the vectorial Maxwell's equation in cylindrical coordinates for the microwire structure; the propagation constant of the hybrid modes can be calculated from the following equation [124], [125]:

$$
\begin{aligned}
{\left[\frac{J_{n}^{\prime}(u)}{u J_{n}(u)}+\frac{K_{n}^{\prime}(w)}{w K_{n}(w)}\right] } & {\left[\frac{J_{n}^{\prime}(u)}{u J_{n}(u)}+\left(\frac{1}{n_{1}}\right)^{2} \frac{K_{n}^{\prime}(w)}{w K_{n}(w)}\right] } \\
& =n^{2}\left(\frac{1}{u^{2}}+\frac{1}{w^{2}}\right)\left[\frac{1}{u^{2}}+\left(\frac{1}{n_{1}}\right)^{2} \frac{1}{w^{2}}\right]
\end{aligned}
$$

where $J_{n}$ is the Bessel function of the first kind, and $K_{n}$ is the modified Bessel function of the second kind. Here, $u$ and $w$ are normalized transverse wave numbers in the dielectric and air region, respectively; calculated as follows:

$$
\begin{aligned}
& u=a \sqrt{\beta_{0}^{2} n_{1}^{2}-\beta^{2}}, \\
& w=a \sqrt{\beta^{2}-\beta_{0}^{2}}
\end{aligned}
$$

where $a$ is the microwire radius, $\beta_{0}$ is the free space propagating constant, and $\beta$ is the propagation constant of the hybrid mode that is calculated by solving (10). Having the propagation constant of modes, one can easily calculate the electromagnetic fields in the dielectric (core) and in the air (cladding) [124], [125]. The normalized electric field component of the fundamental mode in cylindrical coordinates of the air-cladding dielectric wire structure in the microwire regime is depicted in Fig. 16(a). The enhanced evanescent field characteristic of the wires made up of glasses (F2, SF6, SF56, and bismuth glass), polymer (PMMA), and diamond can be seen for the diameters less than the operating wavelength $(\lambda=600 \mu \mathrm{m}$, $f=0.5 \mathrm{THz}$ ).

Table 4 indicates the $\mathrm{PbO}$ concentrations in F2, SF6, and SF57 supplied by Schott-AG and the $\mathrm{Bi}_{2} \mathrm{O}_{3}$ concentration in the bismuth glass supplied by Asahi Glass that has a similar composition to BI-3 glass [127]. The references in Table 4 are the sources of these concentration values, with the exception of SF57 that was obtained by direct measurement using energy dispersive $\mathrm{X}$-ray (EDX) spectroscopy.

To obtain more information about the power distribution in the radial direction, the power fraction outside the core is calculated [123]. Fig. 16(b) shows the power fraction outside the wire versus the fiber diameter. For diameters less than the operating wavelength

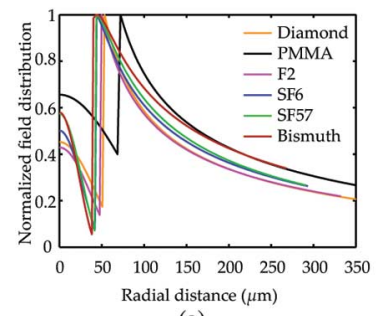

(a)

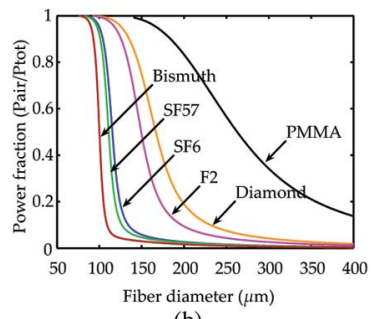

(b)
Fig. 16. (a) Normalized electric field distribution of diamond, PMMA, F2, SF6, SF57, and bismuth glass microwires versus fiber diameter at $\mathrm{f}=0.5 \mathrm{THz}(\lambda=600 \mu \mathrm{m})$. The fiber diameters of diamond, PMMA, F2, SF6, SF57, and bismuth glass microwires are 101.4, 133.2, 87.2, 80.1, 75.3, and 75.4 $\mu \mathrm{m}$, respectively. (b) Power fraction outside the diamond, PMMA, F2, SF6, SF57, and bismuth glass microwires versus the diameter at $\mathrm{f}=0.5 \mathrm{THz}(\lambda=600 \mu \mathrm{m})$. Adapted from [123].

$(\lambda=600 \mu \mathrm{m}, f=0.5 \mathrm{THz}$ ) the power fraction converges to unity. This can be explained in this regime, where the diameter is less than the wavelength, by the fact that most of the guided power is outside the fiber. From Fig. 16(b), it can be concluded that the diameter where the fibers enter the microwire operating regime as well as the slope of the convergence of the power fraction strongly depends on the refractive index of the fiber material [123].

In the microwire operating regime, the fraction of power outside is more than the fraction of power inside, therefore the material absorption has less effect on the loss. For these wires a new description of material absorption called effective loss - the average of the loss coefficients inside (dielectric) and outside (air) the fiber over the transverse field distributions - is used, which is given by [119], [128]

$$
\alpha_{\text {eff }}=\frac{\sigma \int_{0}^{a} \int_{0}^{2 \pi}|E|^{2} r d r d \phi}{\left|\int_{0}^{a} \int_{0}^{2 \pi} S_{z 1} r d r d \phi+\int_{a}^{\infty} \int_{0}^{2 \pi} S_{z 2} r d r d \phi\right|}
$$

where $S_{z 1}$ and $S_{z 2}$ are the $z$-component of the Poynting vectors inside and outside the dielectric wire, respectively. Here, $\sigma$ is the conductivity, related to the material absorption coefficient [119].

The calculated effective loss of the microwires is shown in Fig. 17(a). As expected, the upper limit of the effective

Table 4 Composition of Heavy Metal Oxide Glasses

\begin{tabular}{|cccc|}
\hline Glass & $\mathrm{PbO}$ & $\mathrm{Bi}_{2} \mathrm{O}_{3}$ & Reference \\
\hline F2 & 19 mol. $\%$ & - & Long et al. [126] \\
SF6 & $40 \mathrm{~mol} \%$ & - & Long et al. [126] \\
SF57 & 43 mol. \% & - & - \\
Bismuth & - & 43 mol. \% & Sugimoto et al. [127] \\
\hline
\end{tabular}




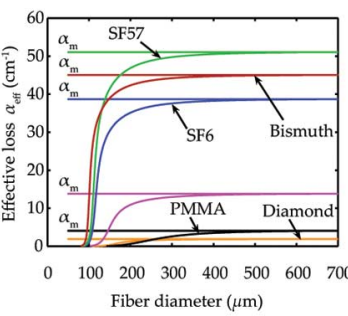

(a)

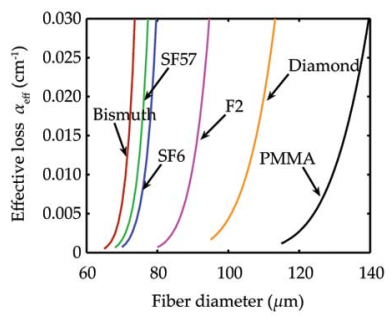

(b)
Fig. 17. (a) Effective loss of fibers made up of diamond, PMMA, F2, SF6, SF57, and bismuth glass materials versus fiber diameter at $f=0.5 \mathrm{THz}(\lambda=600 \mu \mathrm{m})$. (b) Magnification of the lower limit of the effective loss shown in Fig. 17(a). Adapted from [123].

loss of different fibers is defined by the bulk material loss. This is where the fiber diameters are at the same order or larger than the T-ray operating wavelength $(\lambda=600 \mu \mathrm{m})$, therefore the power is confined in the fiber and is encountered with the material loss. For fiber diameters well below the T-ray operating wavelength (T-ray microwire operating regime), the effective loss of all five fibers approaches approximately the same value. To have a clear view of this, Fig. 17(b) shows a zoomed-in view of the effective loss for small diameters, all approaching less than $0.01 \mathrm{~cm}^{-1}$ independently of the material. This can be explained by considering that in this regime the field distribution, although lightly guided through the fiber, has the same or a larger amplitude at the dielectric-air interface, resulting in a larger fraction of guided power outside the fiber. Therefore, the T-ray field around the microwires will be susceptible to atmospheric attenuation, which is dependent on frequency [129], [130]. This work motivates future studies using subwavelength holey fibers, where stronger confinement is expected.

\section{T-RAY NEAR-FIELD IMAGING}

The idea of seeing objects on a finer dimension than what is observable through human eyes has always been intriguing, and its realization has always opened up new lines of fundamental scientific enquiry. Typical human cell sizes range from a few to hundreds of micrometers, depending on the type [131], while bacterial spores like bacillus anthracis (anthrax) are in the order of a few micrometers [132]. The significance of new forms of microscopic analysis is heightened as humankind seeks to probe the nanobio regime. In 1953, Frits Zernike won the Nobel Prize for optical phase-contrast microscopy and, in 1986, Gerd Binnig and Heinrich Rohrer won it for scanning tunneling microscopy (STM) and Ernst Ruska for electron microscopy - these techniques all provided new ways to probe materials and opened up new fundamental science. By bringing noninvasive chemical recognition down to cellular dimensions, near-field T-ray imaging will open up novel applications and new lines of enquiry. One of the current major limitations with T-ray images is the low spatial resolution, as determined by Rayleigh's criterion with a comparatively longer wavelength $(0.3 \mathrm{~mm}$ at $1 \mathrm{THz})$. In an effort to improve the resolution of T-ray images, various techniques have been proposed in the literature with an aim of breaking the diffraction limit. Yuan et al. [133] present a review on nearfield imaging techniques that encompasses the principle of operation and the achieved spatial resolution. However, pulsed T-ray near-field imaging is a rapidly growing area and some important work has been conducted since. In this section, we present a review of the different T-ray near-field techniques in the literature that can broadly be classified into three groups: i) aperture; ii) tip; and iii) highly focused beam methods.

\section{A. Aperture-Based Techniques}

Pulsed T-ray near-field imaging was first demonstrated by Hunsche et al. [134] with an elliptical subwavelength aperture at the end of a tapered metal tip to achieve a spatial resolution of approximately $50 \mu \mathrm{m}(\lambda / 4)$ in illumination mode. A collection mode probe, Fig. 18, operating on a transparent substrate with a metallic aperture has also been demonstrated with a spatial resolution of approximately $40 \mu \mathrm{m}(\lambda / 15)$ [135] and $7 \mu \mathrm{m}(\lambda / 85)$ with $0.5 \mathrm{THz}$ pulses [136].

In this near-field approach, the resolution is no longer determined by the wavelength but by the aperture size. Because much of the radiation is reflected at the aperture plane, the transmitted energy through the subwavelength aperture of size $d$ decreases by $d^{3}$ [137], thereby significantly deteriorating the signal-to-noise ratio for small

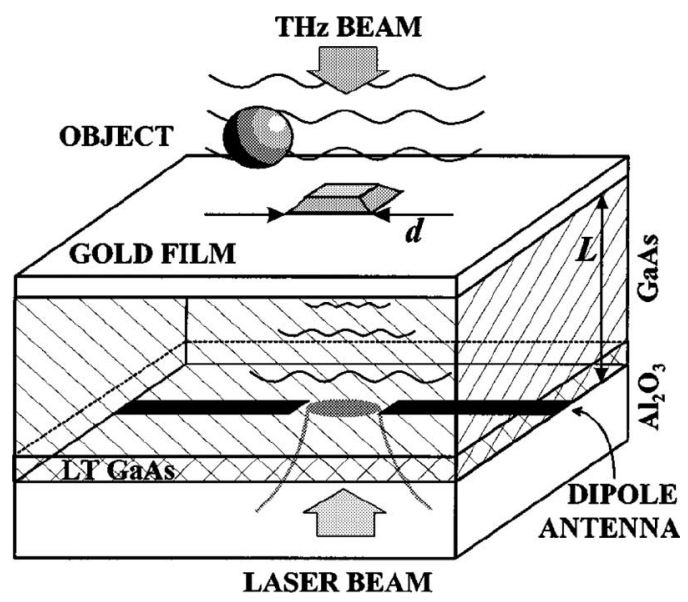

Fig. 18. An example of the aperture-based technique is the collection mode probe presented by Mitrofanov et al. [136]. The aperture is $5 \mu \mathrm{m}$ with a sample separation distance of approximately $2 \mu \mathrm{m}$. The probe achieved a resolution of $7 \mu \mathrm{m}$ with $0.5 \mathrm{THz}$ pulses in the edge test. After Mitrofanov et al. [136]. 
apertures. Low throughput is therefore the major limitation to aperture-based methods. The subwavelength apertures also alter the temporal and spectral content of the transmitted pulses which pose further limitations [137]. Such a method is also difficult to realize in practice as it heavily relies on microfabrication techniques. Another aperture-based technique achieves a subwavelength resolution by means of a dynamic aperture. The technique draws inspiration from the work conducted in the mid-IR frequency range [138] that achieves the aperture effect by modulating the optical beam in a T-ray beam spot on a semiconductor sample. The spatial resolution is determined by the optical beam focal size and a resolution of approximately $\lambda / 10$ is reported [139]. The drawback is low throughput and the need for sample preparation on semiconductor [133].

A recent development of a low-loss dielectric material waveguide with partial metalized sharpened pyramidal tip (Fig. 19) has shown resolution of about $20 \mu \mathrm{m}$ at $80 \mathrm{GHz}$ $(\lambda / 200)$ [140]. The waveguide operates in contact with the sample under study in both illumination and collection mode that does not involve evanescent waves. The micrometer-sized plane facet at the tip end confines the T-rays onto a subwavelength spot and the reflected signal is detected by electrooptic sampling at the other end. The work has been applied to the spectroscopy of dielectric materials [141].

\section{B. Tip-Based Techniques}

Much of the pulsed T-ray tip-based method draws its inspiration from work conducted using infrared radiation [142], [143] and scanning near-field optical microscopy (SNOM) [144]. In the setup, a very sharp solid metallic tip at the end of a metallic probe is dithered near the sample surface illuminated by a far-field T-ray beam. The metallic tip interacts and scatters the evanescent $\mathrm{THz}$ field from the near-field region of the sample for remote detection. A lock-in amplifier along with a T-ray detector is then used to measure the $\mathrm{THz}$ field at the probe oscillation frequency.

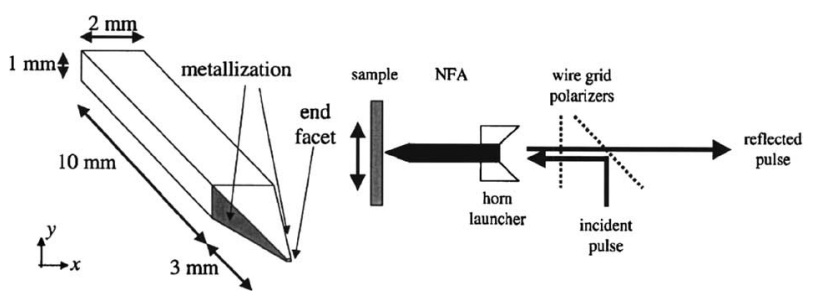

Fig. 19. The low-loss dielectric waveguide with a pyramidal tip achieved a promising resolution of $20 \mu \mathrm{m}$ at $80 \mathrm{GHz}$ [140]. The waveguide operates in contact with the sample. T-ray pulses are coupled onto the waveguide by the horn launcher and the reflected pulses are detected using electrooptic sampling. After Klein et al. [140].

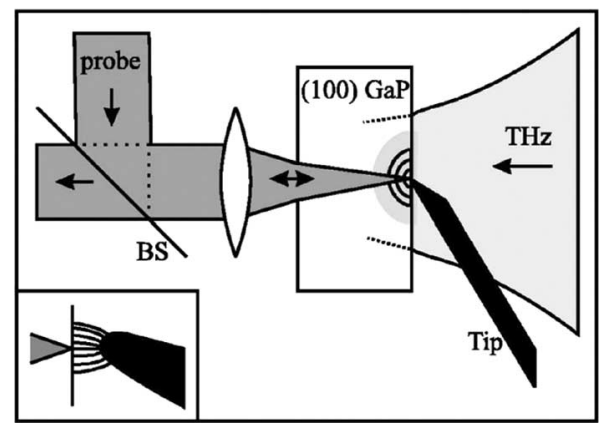

Fig. 20. A tip-based method with electrooptic detection achieved a resolution of $18 \mu \mathrm{m}$ [145]. This technique places the sample in close proximity to the detector (electrooptic or semiconducting medium). A synchronised probe pulse is focused into the crystal and the polarization is changed by the electrooptic effect at the tip, and reflects back for detection. After van der Valk and Planken [145].

In this approach, the tip size determines the T-ray interaction area, which determines the spatial resolution.

The first T-ray tip-based method (Fig. 20) demonstrated a resolution of approximately $18 \mu \mathrm{m}(\lambda / 110)$ [145] with the resolution being determined by the probe laser spot size. A similar setup is implemented with a sphere instead of a tip [146] for near-field measurements.

Another T-ray tip-based method similar to an SNOM setup, mechanically oscillates a copper tip (head diameter $5 \mu \mathrm{m})$ in the near-field region of the sample illuminated by a T-ray beam [147]. The scattered T-rays are detected near the sample surface using a PCA [133], [147]. A resolution of $150 \mathrm{~nm}$ for T-ray pulses is achieved with a similar adaptation of the SNOM setup shown in Fig. 21 [148] Recent reports on implementing atomic force microscopy in conjunction with THz-TDS achieved a resolution of $80 \mathrm{~nm}$ [149].

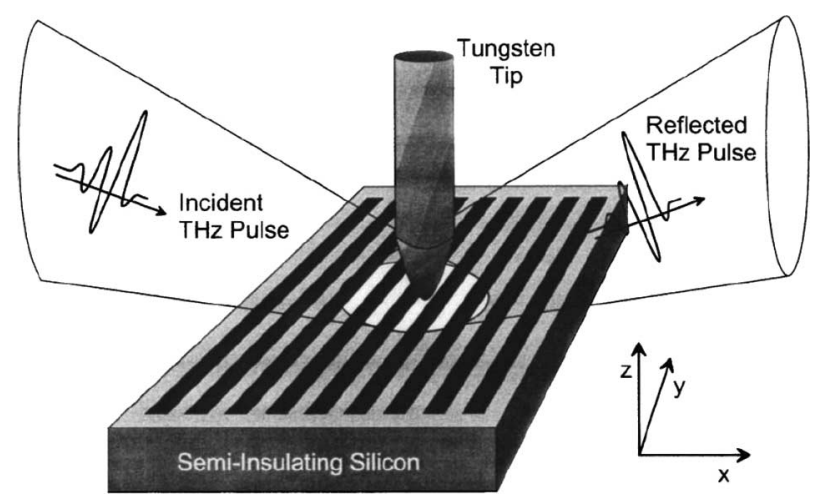

Fig. 21. Another tip-based method [148] achieved a resolution of $10 \mu \mathrm{m}$. The tip has a radius of $100 \mathrm{~nm}$ placed $200 \mathrm{~nm}$ above the sample surface and the transmitted $T$-ray radiation is detected by electrooptic sampling or bolometer. After Chen et al. [148]. 
Instead of illuminating the sample with the generated T-ray radiation, modulating a metal tip in close contact $(<100 \mathrm{~nm})$ to a semiconductor surface in a weakly focused pump laser spot achieved a sub-100 nm spatial resolution [150]. In this method, T-ray radiation is generated by the a semiconductor sample.

The tip-based near-field approach shows great promise in high-resolution surface topographic and T-ray spectral imaging. However, tip use is complex and suffers from low output SNR due to low input power and the scattering at the sample's surface. Furthermore, some techniques are only applicable to semiconductor samples. Chen et al. [148] demonstrated the need to further refine the existing spherical scatter model for the imaging tip [142] and the inclusion of antenna properties for the probe that are discussed in [151], [152]. A possible starting point for future work can be found in Wang and Mittleman [112].

\section{Highly Focused Beam Techniques}

An alternative apertureless near-field imaging technique achieves subwavelength resolution by exploiting the smaller T-ray emission point than the wavelength. In a similar setup to [150] but without the tip, the laser T-ray emission microscope (LTEM) [154] achieved a resolution below $3 \mu \mathrm{m}$ [155] for inspecting electrical faults in integrated circuits. Instead of relying on a tip, the laser pulse is focused to a spot size of about $2.5 \mu \mathrm{m}$ [155] with the backscattered T-rays focused for detection by a bow-tie antenna. Using electrooptics, an optical beam is tightly focused onto a small spot in an optical rectification crystal ( $\mathrm{ZnTe}$ ) to generate a subwavelength diameter T-ray beam in the near-field region [156]. The achievable resolution would be $\lambda / 4.3$, provided the sample is placed on the crystal [156]. Yuan et al. [153] tightly focuses an ultrafast laser to a small spot with an aim of generating T-rays with a spatial resolution close to the laser spot size. The setup in both transmission and reflection mode is shown in Fig. 22. Very thin crystals $(16 \mu \mathrm{m})$ are, however, observed to generate higher powered T-rays at the point of focus than the thicker counterpart $(250 \mu \mathrm{m})$, which reduces the signal [153]. The unexpected phenomenon is shown in Fig. 23. The strong peak from the thin crystal is counterintuitive as thicker crystals provide a greater volume for optical rectification. Furthermore, the signal is linearly dependent on the pump power, provided it is less than $15 \mathrm{~mW}$. The intensity of the pump beam, however, is limited by the two-photon damage threshold of the EO crystal. A thickness in the order of tens of $\mu \mathrm{m}$ is essential to achieve a high resolution. This thickness in turn imposes physical constraints on experimentation and possible future application as thin crystals are thermally and mechanically fragile. A spatial resolution of $20 \mu \mathrm{m}$ is reported [133].

Similar work is presented recently with a $500 \mu \mathrm{m}$ thick crystal for demonstrating a resolution of approxi-

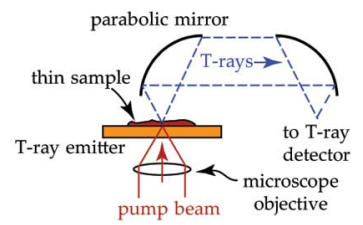

(a) Transmission mode

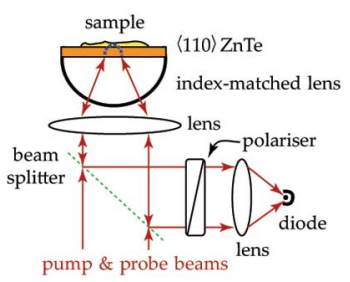

(b) Reflection mode
Fig. 22. Schematics of the electrooptic T-ray microscope in transmission and reflection mode respectively. After Vuan et al. [133]. (a) Transmission mode. (b) Reflection mode.

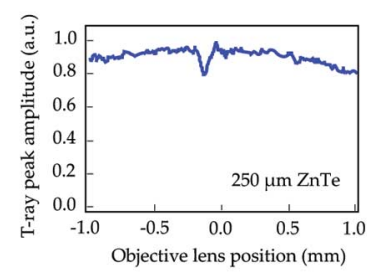

(a) Thick crystal

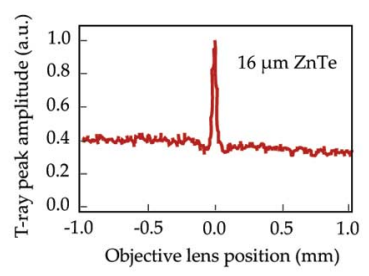

(b) Thin crystal
Fig. 23. Experimental result of Yuan et al. [153]. A thin crystal gave rise to an unexpected enhancement in the emitted $T$-ray power compared to the thick crystal at the focal point of the objective lens. Outside the focal point however, the emitted T-rays of both crystals appear to be constant. After Yuan et al. [153]. (a) Thick crystal. (b) Thin crystal.

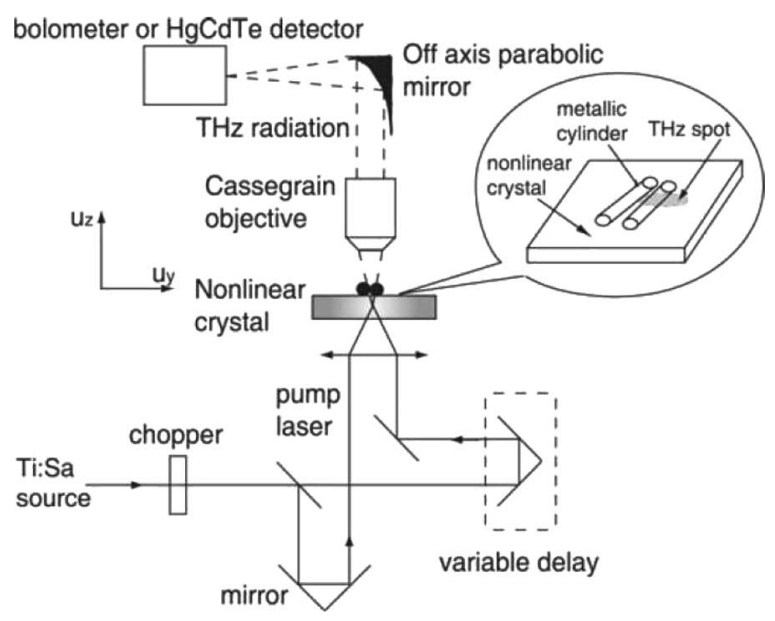

Fig. 24. The electrooptic experimental setup that achieved $75 \mu \mathrm{m}$ resolution with a $500 \mu \mathrm{m}$ thick ZnTe [157]. After Lecaque et al. [157].

mately $75 \mu \mathrm{m}$ [157] as shown in Fig. 24. The resolution is reported to be equal to the size of the focused pump beam. The advantage of the electrooptic methods is throughput and waveform independency of the sample topography [133], [157]. 


\section{Future Directions}

Even though the tip method achieves the highest spatial resolution, the radiation throughput suffers from a reduction of $r^{6} / \lambda^{4}$ with decreasing tip size [158]. This hence places a limitation on the spatial resolution. Contrary to the aperture and tip method, the electrooptical approach is the more efficient alternative with the T-ray power decreasing proportionally to the second power of the source dimension [159]. To date, limited research has been carried out in this area of T-ray near-field imaging. It is widely known that crystal thickness will affect the conversion efficiency, the generated T-ray bandwidth, and the Fabry-Pérot reflections. Existing optical rectification models with a highly focused pump beam [159]-[161] do not address crystal thickness, therefore work is currently underway to investigate the unexpected signal enhancement from thin crystals. In another potential approach, we will place the sample at the end of a customized T-ray waveguide based on the techniques of Atakaramians et al. [122]. The expected resolution is, however, limited by the minimum achievable dimension of the waveguide, which is expected with the current waveguide design to be in the order of $100 \mu \mathrm{m}$. Note that a similar waveguide with a micrometer-sized facet has demonstrated a resolution of $20 \mu \mathrm{m}$ [140], which unlike the tip method, demonstrates a greater power efficiency. Future work will need to model laser heating effects in the thin crystal layer-we propose to extend our previously developed laser heating model [162] that takes into account the proper absorption profile of photons in the material (i.e., an exponential decay with penetration depth).

\section{ALTERNATIVE T-RAY DATA RENDERING}

In order to encourage a rapid adoption of T-ray imaging in industry and medicine, T-ray data should be presented in ways that are useful to professionals in these fields. Established image processing methods can be adapted to T-ray data to enhance its representation. Fig. 25(a) shows an example of a T-ray time-domain image captured by a conventional charge-coupled device (CCD) camera after optical upconversion. This image of a dried insect on a dried leaf consists of $(N \times P)$ pixels and is one time instance along a discretized time domain with $M$ points. By stacking many of these $(N \times P)$ arrays over time, a 3-D time-domain array is obtained.

The time-domain response of one pixel can be extracted for analysis as shown in Fig. 25(b). In Fig. 25(c) we see the time response of three pixels from the leaf/insect specimen mentioned above. The pixels are of the insect, vein of the leaf, and free space. When compared to the free space plot (solid-circle plot), the T-ray pulses after exiting the insect (solid line plot) and leaf (dashed plot) are attenuated in amplitude; this is expected since T-rays are strongly absorbed by moisture. The T-ray pulse through

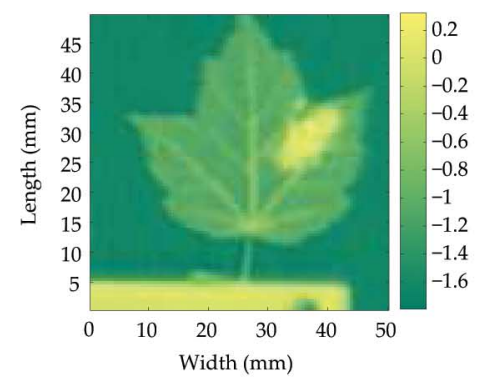

(a)

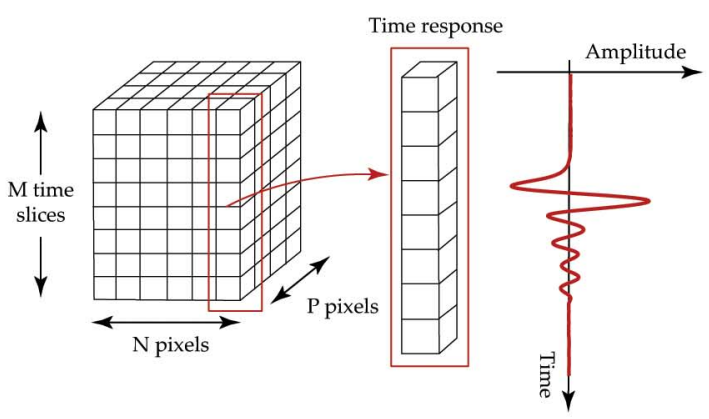

(b)

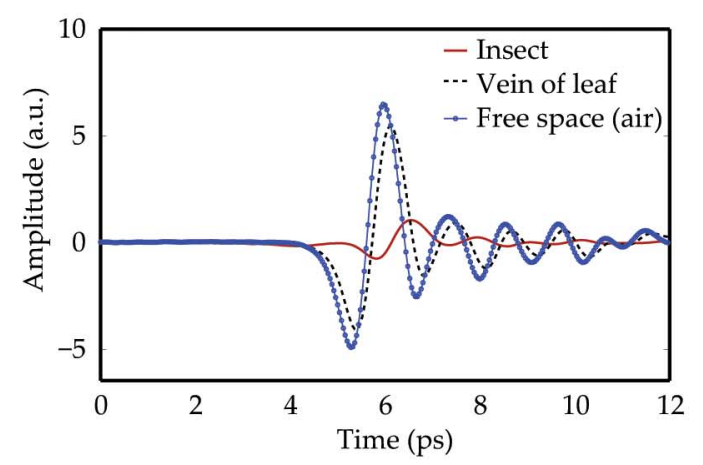

(c)

Fig. 25. (a) Original time-domain image at one time slice (courtesy X.-C. Zhang). (b) Extracting the time response of one pixel from the 3-D time-domain array. (c) The time response of three different pixels: insect, vein of leaf, and free space.

the insect is phase shifted more than that through the leaf, implying that the insect is thicker than the leaf and/or has a higher refractive index. These time-domain waveforms can be Fourier transformed to obtain the broadband frequency response.

Fig. 26(a) shows T-ray data after application of common image processing techniques, such as edge detection, Laplacian filtering, and smoothing. Instead of separate amplitude and phase plots, this enhanced image more closely resembles 3-D images generated using medical visualization tools, thus allowing medical professionals to analyze and compare results easily.

An open question is: can we implement a new form of T-ray data representation inspired by established image 


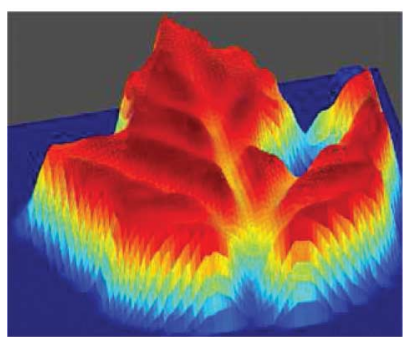

(a)

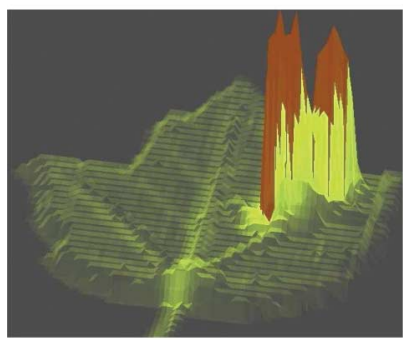

(b)

Fig. 26. (a) Improvements made to T-ray image after application of common image processing techniques. The third dimension (z-axis), intensity, is used to pseudocolor this image. (b) With T-ray pseudophase contrast imaging. The third dimension ( $z$-axis) in this case is thickness. After [165].

enhancement techniques used in the scientific field, such as Zernike's optical phase contrast method? Zernike's optical phase contrast method is based on the effect of light passing through a phase object. ${ }^{2}$ When light passes through free space/air (surround path), the phase and amplitude information in the light wave is unaltered. When light passes through a phase object (particle path), the amplitude of the light wave is slightly attenuated when compared to the surround path. The light wave also slows down due to the refractive index of the phase object; the amount of delay introduced will also depend on the object's thickness. There is a phase difference between the surround and particle paths. The human eye can only sense amplitude and color differences when both the surround and particle paths are in phase. The eye, however, fails to sense the contrast between the surround and particle paths due to their being out of phase [164]. Phase objects therefore appear invisible under a conventional light microscope. In optical phase contrast, the surround path is manipulated to bring it back in phase with the particle path, resulting in the visibility of the phase object due to constructive interference.

\footnotetext{
${ }^{2} \mathrm{An}$ object that is not observable (transparent, invisible) because it does not provide contrast with the background. It does, however, cause phase modulations in the propagating light wave, whereby these phase modulations are due to either the different refractive index of the object from air, the thickness of the object, or both [163].
}

Considering one pixel from the leaf/insect sample in this study, the path length of the sample can be obtained indirectly by first calculating the relative time delay between the peak of the samples (e.g., at position $i$ ) and the peak of a reference signal. Repeating for the whole $N \times P$ matrix, the relative propagation times $\tau_{i}$ with respect to $\tau_{\text {ref }}$ is given by

$$
\begin{aligned}
& \tau_{i}=\mid(\text { time occurrence of peak } i)-\tau_{\text {ref }} \mid \\
& \text { for } i \epsilon 1, \cdots, N \times P .
\end{aligned}
$$

The optical path length is then given by

$$
d_{i}=\text { speed of light } c \times \tau_{i} \text { for } i \epsilon 1, \cdots, N \times P \text {. }
$$

The optical path lengths can now be converted to sample thicknesses. In Fig. 26(b), thickness is represented by the vertical dimension - the $z$-axis - thus making the image appear three-dimensional. There are strong contrasts between the different parts of the leaf, and between the two halves of the insect. The various path lengths have been translated into an image with high contrast, thus a novel form of phase contrast has been achieved.

In addition to rendering thickness, opacity provides an additional slice of information about a sample's characteristics. To calculate opacity, a reference pixel is first identified. This pixel is a free space pixel with peak temporal amplitude $I_{\text {ref. }}$. The peak temporal amplitude of the sample pixels are then subtracted from $I_{\text {ref }}$ as follows:

$I_{i}=\mid I_{\text {ref }}-($ peak amplitude at pixel $i) \mid$

$$
\text { for } i \epsilon 1, \cdots, N \times P \text {. }
$$

This difference tells us how opaque a sample is. If $I_{i}=I_{\text {ref }}$, then there is zero opacity (free space is transparent). If $I_{i}=\max \left[I_{i \in 1, \ldots, N \times P}\right]$, then there is unity opacity (opaque). The opacity of each pixel can now be set so that the final rendered image shows both thickness and opacity as one would see in the real world. Pseudocoloring can also be applied as in Fig. 26(b) to make the image look more realistic.

The low SNR, however, makes it difficult to pin-point a particular pixel as the reference. To overcome this, peak detection was performed on every pixel in the time domain, i.e., the peak of each $M \times 1$ array was found. The peak detection extracted from each pixel the time occurrence and value of the peak amplitude, thus generating two $N \times P$ matrices. From each matrix, 15 neighboring pixels that correspond to the top left corner of Fig. 25(a) were selected and used to average $I_{\text {ref }}$ and propagation time $\tau_{\text {ref }}$. 
The leaf used in this investigation is dry, thus it has minimal water content; strong water absorption that causes deformation of the profile is not expected. Internal reflection is ignored. The insect and the portion of leaf beneath it are treated as one object. Although the insect is a dried specimen, it is thicker and/or denser than the leaf, thus some pulse broadening in the T-ray profile is apparent. This is seen by the solid line plot in Fig 25(c). When the time profiles of the leaf, insect, and free space are compared, it is assumed that all three time profiles have the same polarity. The positive peaks from all three profiles are therefore used wherever peak temporal amplitudes are involved in this investigation.

Note that Zernike's original phase contrast method is based on interference between two beam paths. By altering the path length of one path, the phase difference between the two paths is revealed proportionally as amplitude variations in the observed image. This is akin to heterodyne detection. However, with functional T-ray imaging, the full phase information is recovered and so interference effects are not exploited. Our method of rendering sample thickness on the z-axis, as shown in Fig. 26(b), is therefore dubbed "pseudophase contrast." Future work could consider signal processing techniques that transform the full phase information from T-ray imaging to produce the actual appearance of Zernike phase contrast images. Given that T-ray functional imaging recovers the full phase information, this should be possible in principle.

\section{3-D T-RAY TOMOGRAPHY}

Tomographic imaging or tomography refers to any method that aims to nondestructively image the internal section of an object. This principle can be found in a wide range of "modalities," including X-ray computed tomography (X-ray $\mathrm{CT}$ ), magnetic resonance imaging (MRI), ultrasonic imaging, etc. [166]. The differences of these modalities are fundamentally inherited from the type (sound or electromagnetic) of wave and the frequency of wave in use, which have different capabilities to penetrate and resolve objects.

Tomography by means of a modern T-ray system was first demonstrated by Mittleman et al. [167] in analogy to ultrasonic B-scan imaging [168], so-called time-of-flight tomography. It allows internal inspection of any object transparent or translucent to T-rays. Shortly after illuminating an object with a broadband T-ray pulse, a series of reflected pulses at refractive-index discontinuities inside an object are collected as a signal trace in the time domain. This enables mapping from the object's internal discontinuities to a refractive-index profile in the direction of propagation. A full 3-D map of the object is obtainable by raster-scanning of an object from only one direction. Due to its simplicity yet efficiency, the T-ray B-scan mode is employed to inspect many targets, for instance, space shuttle foam insulation [169], pharmaceutical coating [170], a hidden aluminum oxide layer under opaque paint [171], etc. However, B-scan images lack quantitative aspects available in computed tomography.

In this section, further achievements on two tomographic imaging techniques based on transmission mode of pulsed T-rays are reviewed. The systems are demonstrated and the achievable resolution and image quality are investigated.

\section{A. T-Ray Computed Tomography (T-Ray CT)}

T-ray computed tomography shares a common principle with X-ray CT, the technology currently hosted in hospitals, airports, and factories. Generally, at a step, a CT scan emits a plane wave and records a series of waves that are transmitted through or reflected from a target in 2-D plane. This step is repeated at different angles around the object. Assuming the received signals are line integrals along the direct paths [172], the Fourier-slice theorem [166] can be applied. Subsequently, the reconstruction process, such as direct Fourier reconstruction [173], filtered backprojection (FBP) [174], or the algebraic reconstruction technique (ART) [175], estimates the entire volume of an object.

The accumulative distortion of waves along their propagation path through an object is proportional to the projection, which equals a line integral of the object function along a line [176]. The projection

$$
p(\theta, l)=\int_{L(\theta, l)} o(x, z) d l
$$

where $\theta$ is the projection angle, and $l$ is the distance from the axis of rotation perpendicular to the ray propagation. The variable $o(x, z)$ is the object function of the target. The integral is calculated over a straight line $L$ joining the source and the detector. This transformation is known as the radon transform. The basic concept is depicted in Fig. 27. In case of T-ray CT, the object function and the projections can be, for example, the attenuation coefficient, the index of refraction, or any related quantities.

To reconstruct the target's object function $o(x, z)$, the filtered backprojection algorithm is used

$$
o(x, z)=\int_{0}^{\pi} q_{\theta}(x \cos \theta+z \sin \theta) d \theta
$$

where

$$
q_{\theta}(l)=\int_{-\infty}^{\infty} P_{\theta}(\nu) \exp (j 2 \pi \nu l) d \nu
$$




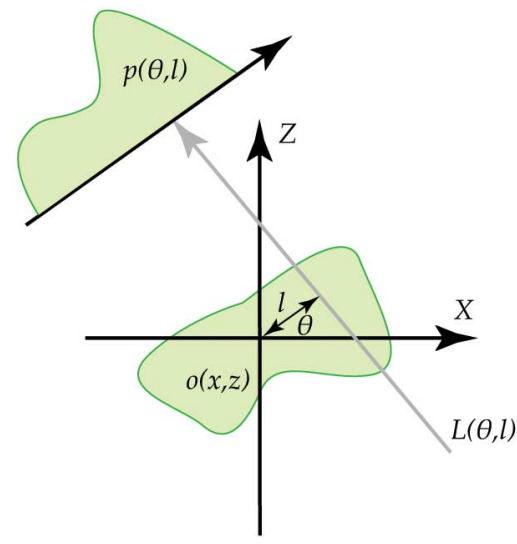

Fig. 27. An object $o(x, z)$ and its projection $p(\theta, I)$. The radon transform defines the projection $p(\theta, l)$ as the line integral over the straight line $L$ of the object function $o(x, z)$. The projection offset $l$ is the perpendicular distance of the projection from the axis of rotation. The projection angle $\theta$ and $x$ and $z$ define a standard rectangular coordinate system.

Equation (19) can be seen as a filtering operation. The filtered projection $q_{\theta}(l)$ is calculated by Fourier transforming $p_{\theta}(l)$, filtering the result using a filter with a frequency response of $|\nu|$ and then inverse Fourier transforming the result. The filtered backprojection algorithm, (18), allows the object function at each value of $(x, z)$ to be reconstructed. For a point $(x, z)$ there is a corresponding value of $l=x \cos \theta+z \sin \theta$ for each projection $\theta$. The value of $o(x, z)$ is recovered by summing the value of the filtered projections at the corresponding $l$ over all angles $\theta$. In the discrete case $l$ may not always be known at the required value and in this case bilinear interpolation is most often used and produces highly accurate results.

In the experiment, a chirped probe pulse T-ray imaging system [177] is used for T-ray CT. The target was mounted on a rotation stage, a $250 \mathrm{~mm}$ focal length parabolic mirror is used to focus the $\mathrm{THz}$ radiation to a spot size of $2 \mathrm{~mm}$ at the target. Parabolic mirrors after the sample are positioned to image the field from a plane near the target onto the ZnTe sensor. To ensure a high SNR, a PCA T-ray source is used. The electrodes are separated by $16 \mathrm{~mm}$ and a bias of $2 \mathrm{kV}$ is applied.

The optical probe pulse is linearly chirped and temporally stretched to a pulse width of $30 \mathrm{ps}$, using a grating pair with a separation of $4 \mathrm{~mm}$, an incident angle of $51^{\circ}$ and a grating constant of $10 \mu \mathrm{m}$. The $\mathrm{THz}$ pulse modulates the probe pulse via the EO effect and is recovered with a spectrometer (SPEX 500M) and CCD camera (PI-Pentamax). The CCD exposure time is set to $15 \mathrm{~ms}$. This allows a $\mathrm{THz}$ pulse to be measured in approximately $60 \mathrm{~ms}$ and provides a signal-to-noise ratio of approximately 100 . The bandwidth of the system is limited

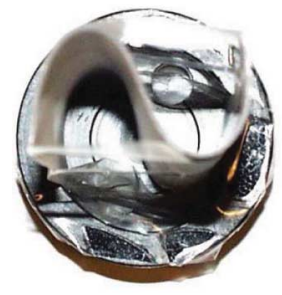

(a)

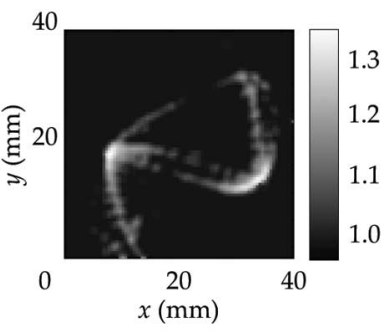

(b)
Fig. 28. (a) Top view of a $0.6 \mathrm{~mm}$ thick polyethylene sheet folded into an "S" shape for CT experiment. The polyethylene was held in place using scotch tape. The sample was mounted such that the T-ray beam propagated in the plane of the paper and the sample was rotated about the $y$-axis (the axis pointing out of the page). (b) A time-domain reconstruction of the sheet of polyethylene. The measured data was reconstructed using the filtered backprojection algorithm. The timing of the T-ray pulses was used as the input to the algorithm.

by the chirped pulse detection technique to approximately $1 \mathrm{THz}$. The CCD resolution results in a sampling period of $0.15 \mathrm{ps}$ and a spectral resolution of $17 \mathrm{GHz}$.

The target shown in Fig. 28(a) consists of a sheet of polyethylene bent into an " $\mathrm{S}$ " shape. The target is imaged at 20 different heights and $10^{\circ}$ angular increments, and the total acquisition time is under $13 \mathrm{~min}$. The time-domain phase delay is estimated. This data is used to reconstruct the cross section of the target by the filtered backprojection algorithm, as shown in Fig. 28(b). The target is reconstructed accurately, and the resolution is sufficient to reconstruct the scotch tape used to hold the polyethylene in place. A 3-D image can be formed by reconstructing each horizontal slice and surface rendering them as illustrated in Fig. 29. The 3-D image is formed by rendering the isosurface formed at $50 \%$ of the peak reconstructed refractive index.

\section{B. T-Ray Diffraction Tomography (T-Ray DT)}

If the object inhomogeneities are comparable in size to the propagation wavelength, diffractions along the

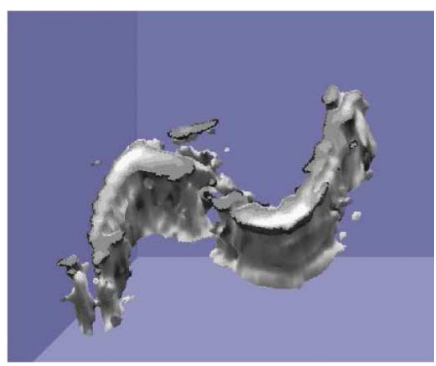

Fig. 29. A 3-D model of the sheet of polyethylene reconstructed from computed tomography and rendered by surface rendering. 
path must be taken into account, and the assumption of a direct line integral is no longer valid. This situation can regularly occur with T-ray tomography, where the T-ray wavelengths are in the order of submillimeters, the common size of target's features. A precise description is to relate the scattered field to the object's spatial distribution via the nonlinear wave equation. However, a linear approximation, either first-order Born or Rytov, is sufficient in most cases. Several reconstruction algorithms based on this Fourier Diffraction projection Theorem [178] are available, e.g. first-order approximation frequency interpolation [179] or filtered-backpropagation [180].

Theoretically, the relationship between the $\mathrm{THz}$ wave distribution and the target's refractive index as a function of position $\mathbf{r}$ can be described by the scalar Helmholtz equation

$$
\Delta^{2} u(\mathbf{r})+k_{0}^{2} \hat{n}(\omega, \mathbf{r})^{2} u(\mathbf{r})=0
$$

where $u(\mathbf{r})$ is the electromagnetic field complex amplitude function, and $k_{0}=2 \pi / \lambda$ is the wave number of the electromagnetic wave in a vacuum.

Our goal is to determine a target's refractive index function $\hat{n}(\mathbf{r})$, given the measured $\mathrm{THz}$ wave amplitude and phase distribution $u(\mathbf{r})$, which is known as the inverse scattering problem (ISP) [181]. Equation (20) may be written as

$$
\left(\Delta^{2}+k_{0}^{2}\right) u(\mathbf{r})=-o(\mathbf{r}) u(\mathbf{r})
$$

where $o(\mathbf{r})$ is the target's object function, given by

$$
o(\mathbf{r})=-k_{0}^{2}\left[\hat{n}(\omega, \mathbf{r})^{2}-1\right]
$$

The solution of (21), $u(\mathbf{r})$, can be considered to be the sum of two components

$$
u(\mathbf{r})=u_{0}(\mathbf{r})+u_{s}(\mathbf{r})
$$

where $u_{s}(\mathbf{r})$ is the scattered field caused by the target and $u_{0}(\mathbf{r})$ is the incident field, the field that would be present without a target, or, equivalently, a solution to the homogeneous Helmholtz equation

$$
\left(\Delta^{2}+k_{0}^{2}\right) u_{0}(\mathbf{r})=0
$$

Combining (24), (23), and (21) yields a wave equation for the scattered component

$$
\left(\Delta^{2}+k_{0}^{2}\right) u_{s}(\mathbf{r})=-o(\mathbf{r}) u(\mathbf{r})
$$

The Rytov approximation offers a linearization of the wave equation. It assumes that the incident wave perturbation caused by the target can be described by a change of phase in the reference wave. It is derived by considering the total field to be represented as complex phase [182]

$$
\begin{aligned}
u(r) & =\exp [\phi(\mathbf{r})] \\
& =\exp \left[\phi_{0}(\mathbf{r})+\phi_{s}(\mathbf{r})\right]
\end{aligned}
$$

Combining (24), (25), and (26) and reducing the resultant expressions [166] yields

$$
\left(\Delta^{2}+k_{0}^{2}\right) u_{0}(\mathbf{r}) \phi_{s}(\mathbf{r})=-u_{0}(\mathbf{r})\left[\left(\Delta \phi_{s}(r)\right)^{2}+o(\mathbf{r})\right] .
$$

The first Rytov approximation assumes that the gradient of the scattered complex phase $\phi_{s}(\mathbf{r})$ is small, therefore

$$
\left(\Delta \phi_{s}(r)\right)^{2}+o(\mathbf{r}) \approx o(\mathbf{r})
$$

The solution for the scattered phase $\phi_{s}(\mathbf{r})$ is then

$$
u_{0}(\mathbf{r}) \phi_{s}(\mathbf{r})=\int G\left(\mathbf{r}-\mathbf{r}^{\prime}\right) o\left(\mathbf{r}^{\prime}\right) u_{0}\left(\mathbf{r}^{\prime}\right) d \mathbf{r}^{\prime}
$$

where the Green's function is the solution of the differential equation

$$
\left(\Delta^{2}+k_{0}^{2}\right) G\left(\mathbf{r}-\mathbf{r}^{\prime}\right)=-\delta\left(\mathbf{r}-\mathbf{r}^{\prime}\right)
$$

The complex phase of the scattered field is therefore given by

$$
\phi_{s}(\mathbf{r})=\frac{1}{u_{0}(\mathbf{r})} \int G\left(\mathbf{r}-\mathbf{r}^{\prime}\right) o\left(\mathbf{r}^{\prime}\right) u_{0}\left(\mathbf{r}^{\prime}\right) d \mathbf{r}^{\prime}
$$

The Rytov approximation requires that the phase of the scattered field varies slowly relative to one wavelength. 


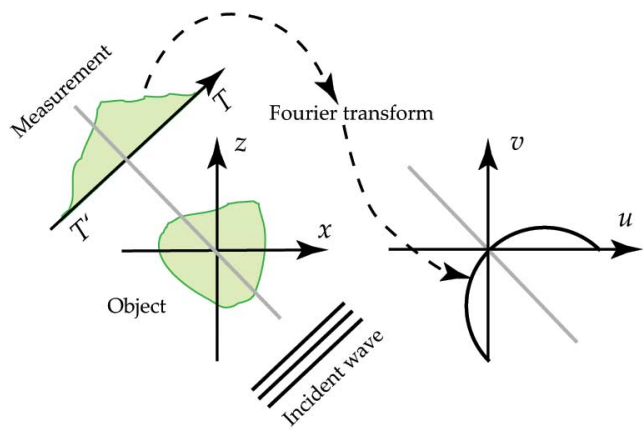

Space domain

Frequency domain
Fig. 30. The Fourier diffraction theorem in two dimensions. The Fourier diffraction theorem relates the Fourier transform of a diffracted projection to the Fourier transform of the object along a semicircular arc. After [166].

These approximations to the Helmholtz equation allow linear reconstruction algorithms to be developed to reconstruct the target's object function $o(\mathbf{r})$ based on measurements of the diffracted radiation from multiple projections.

The reconstruction was performed in the frequency domain, by Fourier transforming the measured T-ray time-domain pulses and using the Fourier coefficients at a single frequency. The solution to this problem is found in the Fourier diffraction theorem, which states that [166]

when an object $o(x, z)$ is illuminated with a plane wave as shown in Fig. 30, the Fourier transform of the forward scattered field measured on the line $\mathrm{TT}^{\prime}$ gives the values of the 2-D transform $O(u, v)$, of the object along a semicircular arc in the frequency domain, as shown in the right half of the figure.

Given the scattered field from a single projection angle, we may determine the spatial Fourier transform of the object function along an arc. However, this alone is not sufficient to determine an accurate estimate of the object function of the target. By rotating the target we obtain the scattered field at different orientations. Each of these provide an estimate of the spatial Fourier transform of the object function along a different arc. The arcs rotate as the target is rotated [183], and by rotating through $360^{\circ}$ the full Fourier space may be populated, up to a maximum frequency of $\sqrt{2} k$.

To acquire the required diffraction data a T-ray diffraction tomography system is developed. The T-ray DT system is based on the 2-D electrooptic imaging system [184] and utilizes synchronized dynamic subtraction and sensor calibration to provide a sufficient SNR. The sample is mounted on a computer controlled rotation stage and positioned $50 \mathrm{~mm}$ from the ZnTe detector. A PCA THz

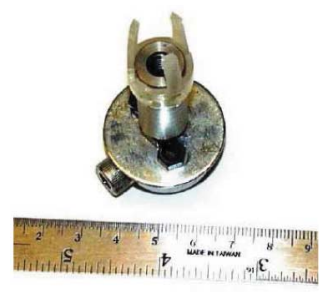

(a)

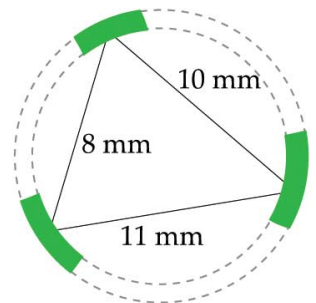

(b)
Fig. 31. (a) A T-ray DT test structure consists of three rectangular polyethylene cylinders. (b) Its geometry. The rectangular cylinders have dimensions of $2.0 \times 1.5,3.5 \times 1.5$, and $2.5 \times 1.5 \mathrm{~mm}$ (clockwise from top).

emitter is used to maximise the THz power and SNR. The PCA used has an electrode spacing of $16 \mathrm{~mm}$ and a bias voltage of $2 \mathrm{kV}$ is applied.

A single motion stage is required to scan the $\mathrm{THz}$ temporal profile and the target is rotated to obtain an image at multiple projection angles. The minimum data acquisition period for a CCD exposure time of $15 \mathrm{~ms}$ per frame and a projection step size of $10^{\circ}$ is approximately $8 \mathrm{~min}$. However, in practice, multiple CCD frames are averaged to improve the SNR.

A complicated sample is constructed to investigate the properties of the system with a Rytov approximation based reconstruction algorithm. The target structure consists of three rectangular polyethylene cylinders. This test structure and its geometry are illustrated in Fig. 31. A quasi-3-D reconstruction may be performed by performing a 2-D reconstruction on each horizontal slice of the measured CCD data. The reconstructed slices may then be combined to form a 3-D image. This is performed for the target shown in Fig. 31 and is illustrated in Fig. 32. Each reconstructed slice is thresholded at $50 \%$ of the peak

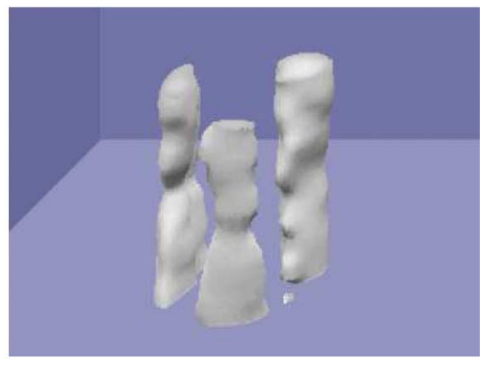

Fig. 32. Reconstructed 3-D image of the polyethylene cylinders. Each horizontal slice was reconstructed independently from diffraction tomography and combined to form a 3-D image. The visible ripples on the surface of the cylinders are a result of the thresholding procedure and are caused by noise in the reconstructions. 
amplitude, the slices are then combined and surface rendered to generate a 3-D image.

In spite of the aforementioned capabilities, T-ray tomography techniques are, by virtue of relatively low system SNR, limited to the laboratory environment, where the acquisition time is long and the target is small [185]. Of late this field is undergoing something of a renaissance as T-ray sources and detectors have developed to a point where high signal to noise ratio and reasonable acquisition rates are possible. One key objective is to combine threedimensional imaging with spectroscopic information capture [186], [187].

\section{WAVELET-BASED LOCAL TOMOGRAPHY RECONSTRUCTION}

With traditional CT techniques as those in Section X, full exposure data are needed for a reconstruction algorithm to produce cross sectional images. For time-domain terahertz measurements, the requirement for full exposure data is impractical due to the slow measurement process. In this section, we apply a wavelet-based algorithm [188] to reconstruct $\mathrm{T}$-ray $\mathrm{CT}$ images with a significant reduction in the required measurements.

The reconstruction algorithm represented in this section recovers the 2-D scaling and wavelet coefficients of an image directly from its projections via a backprojection algorithm. It reduces the calculation time when wavelet coefficients, rather than complete data, are applied. In addition, a good approximation of the image in the region of interest (ROI) is achieved via the use of image projections on lines intersecting the local ROI, plus a small number of projections in the immediate vicinity.

The algorithm is summarized as follows.

- The original projections are calculated from the measured desired parameters of T-ray imaging.

- The region of exposure is truncated for the reconstruction of an image only in the region of interest.

- The region of exposure of each projection is filtered by modified wavelet and scaling filters at all projection angles to find wavelet- and detailcoefficient projections, respectively.

- The filtered projections at the previous step are extrapolated to delete artifacts along the borders of the ROE in the projections.

- The extrapolated projections are filtered by scaling and wavelet ramp filter and then backprojected to obtain the approximate and detail cross section.

In order to validate local reconstruction of T-ray measurement using the proposed algorithm, the target sample of a polystyrene cylinder with holes drilled, Fig. 33(a), is investigated. The sample is measured at 25 projection angles along $180^{\circ}$ projection area, with 101 projections at each of projection angle. The measured spatial interval is $0.5 \mathrm{~mm}$.

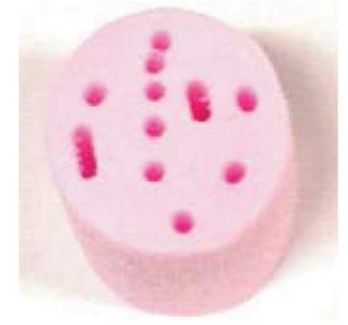

(a)

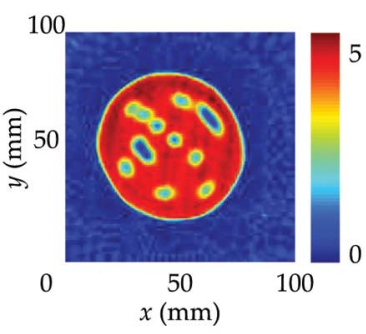

(b)

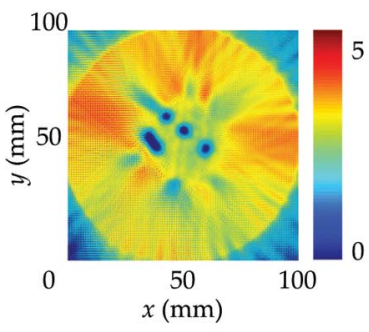

(c)
Fig. 33. (a) Detailed polystyrene resolution test target, drilled with 2 mm diameter holes of varying interhole distances. (b) A global reconstruction of a polystyrene cylinder with holes using a traditional FBP algorithm. (c) A wavelet-based local reconstruction.

The wavelet-based reconstructed image is shown in Fig. 33(c). Notice that a centered disk radius of 16 pixels of the polystyrene phantom can be recovered from the extrapolated scaling and wavelet coefficients using local reconstruction method, with $46 \%$ of full projection data. For comparison, Fig. 33(b) illustrates the global reconstruction, using traditional FBP algorithm. It is shown that our algorithm allows a satisfactory local reconstruction with well-defined local features in the region of interest.

In conclusion, we have developed an algorithm to locally reconstruct a cross section of an object from T-ray projection measurement with use of wavelet and scaling coefficients. The local reconstruction scheme is effective for recovery of a cross section of a target object with local data from a global T-ray CT measurement.

\section{T-RAY SUBSTANCE RETECTION}

T-rays have proven to be of great use in the detection of biological and nonmetallic substances that are not readily detected by other means. In many instances, conventional methods of retection (detection of hidden objects or substances within a package), such as X-rays manage only to reveal dense objects, but have difficulties in detecting plastic objects or soft biological materials. T-rays are able to improve on X-rays, due to their ability to detect nonmetallic and nonpolar substances, as well as offering spectroscopy of these substances. T-ray 


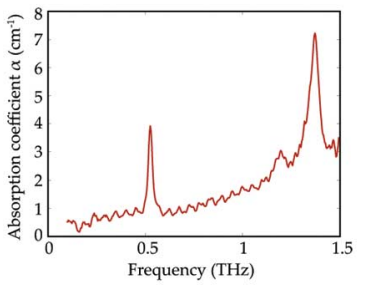

(a)

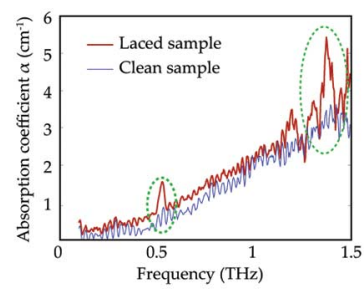

(b)
Fig. 34. (a) T-ray absorption spectrum of $\alpha$-lactose monohydrate at room temperature from 0.1 to $1.5 \mathrm{THz}$. Distinct peaks can be seen at approximately 0.52 and $1.38 \mathrm{THz}$, which are unique to $\alpha$-lactose, showing its distinct T-ray signature. In comparison, (b) shows the T-ray spectrum of a nylon cotton sandwich, with both a clean sample (blue plot) and an $\alpha$-lactose laced sample (red plot) at room temperature. Two sheets of nylon, each with a thickness of $3.205 \mathrm{~mm}$, were pressed together with a sheet of cotton $(0.442 \mathrm{~mm}$ thick) in between in order to crudely simulate the profile of a plastic suitcase. The resultant spectra for the clean sample shows no signature absorption peaks. Absorption of the T-ray signal is increased with increasing frequencies, as the nylon and cotton absorb the signal to a greater degree. Signal noise and interface reflections can also be seen in the data. The laced sample clearly shows the signature absorption peaks from Fig. 34(a) when the cotton is laced with $\alpha$-lactose. The circled areas clearly show the absorption peaks at approximately 0.52 and $1.38 \mathrm{THz}$, which are characteristic to $\alpha$-lactose, indicating that it is present in this particular sample.

imaging has shown this to be possible in detection of objects hidden under clothing and within envelopes [29], [189]-[193].

T-ray spectroscopy can also be used to discern differing substances, due to their varying absorption peaks across the terahertz frequencies that are unique to each substance. This leads to a readily identifiable signature for that substance. Previous experiments have shown that T-ray spectroscopy is able to detect biological agents [29], [189], [190], explosives [191], [192], and narcotics [193], as they have distinct terahertz signatures.

In a proof-of-concept experiment, it is shown that T-rays are able to penetrate various common plastics and detect a trace substance laced on a piece of cotton wedged between them [194]. The experiment uses $\alpha$-lactose, which has distinct terahertz absorption peaks at approximately 0.52 and $1.38 \mathrm{THz}$ [Fig. 34(a)]. The experiment shows that when a small amount of lactose is laced onto cotton fabric, and then sandwiched between two sheets of the same plastic, high impact polystyrene (HIPS), lexan, nylon, glycol modified polyethylene terephthalate (PETG), polycarbonate, or polyethylene, the absorption peaks are still readily detectable [Fig. 34(b), red plot]. This is opposed to a similar lactose-free setup, where no absorption peaks are seen [Fig. 34(b), blue plot].

The two plastic sheets surrounding the cotton appear only to attenuate the absorption peaks and add interface reflection noise shown in Fig. 34(a) and (b). This shows that other organic and nonmetal substances may be detectable under similar circumstances.

All tested plastics are found to be transparent under T-ray spectroscopy, only attenuating the signal to a slight degree. Nylon appears to be the most transparent under T-rays, while PETG attenuates the T-ray signal to the highest degree of the plastics tested. Absorption coefficients at $0.8 \mathrm{THz}$ were $0.400,0.515,1.500,3.755,6.400$, 6.955 , and $12.200 \mathrm{~cm}^{-1}$ for nylon, polyethylene, HIPS, cotton, lexan, polycarbonate, and PETG respectively. ${ }^{3}$

The results from these experiments can lead to further research into the application of T-rays in use for scanning of packages and luggage in secure areas to readily and actively detect harmful or dangerous organic substances. Clothing within luggage or a package may also be screened to determine if a trace substance is present for potential quarantine applications.

\section{CONCLUSION}

In this paper, we have presented selected T-ray research topics that have been investigated by the Adelaide T-ray group. The topics - in terms of core hardware-include an uncertainty analysis of THz-TDS systems, doubled-modulated DTDS for liquid spectroscopy, microwires for T-ray transmission, and T-ray near-field imaging techniques. For molecular and biological applications, the topics encompass vibrational mode prediction, bioaffinity sensing, and T-ray propagation in multilayered media. Beyond onedimensional spectroscopy, the topics extend to functional imaging techniques - including image rendering, CT and DT tomography, and rapid local tomographic reconstruction with wavelets. As a promising application of T-rays, substance retection is another ongoing topic.

\section{Acknowledgment}

The authors gratefully acknowledge a number of coworkers and collaborators during the course of this work: Shahraam Afshar Vahid, Douglas A. Gray, Gregory Metha, Tanya M. Monro, Jesper Munch, Tamath J. Rainsford. Thanks are due to David N. Jamieson for supplying the diamond samples, Finlay Shanks for assistance with midIR measurements, Alban O’Brien for laboratory technical assistance, Heike Ebendorff-Heidepriem for carrying out the EDX concentration measurement of SF57, and Asahi Glass Company for supplying the bismuth glass sample. A very warm thanks is due to X.-C. Zhang, and all his group, who have closely collaborated since the inception of our group in 1997. The authors are greatly indebted to X.-C. Zhang for seeding our group in the early days- - he is the "father of T-rays."

\footnotetext{
${ }^{3}$ The absorption coefficients of materials shown in this section are different from the values reported in Section IV, as they are from different suppliers - variation in additives can heavy impact on the properties of plastics [195].
} 


\section{REFERENCES}

[1] L. C. Robinson, "A survey of methods of generating millimeter and sub-millimeter electromagnetic waves," Weapons Research Establishment, Australian Defence Scientific Service, Dept. Supply, Tech. Rep. ERD 57, 1958.

[2] P. H. Siegel, “Terahertz technology," IEEE Trans. Microw. Theory Tech., vol. 50, no. 3, pp. 910-928, Mar. 2002

[3] P. H. Siegel, “THz technology: An overview," Int. J. High Speed Electron Syst., vol. 13, no. 2, pp. 351-394, 2003.

[4] M. C. Nuss, "Chemistry is right for T-ray imaging," IEEE Circuits Devices Mag., vol. 12, no. 2, pp. 25-30, Mar. 1996.

[5] G. Mourou, C. V. Stancampiano, A. Antonetti, and A. Orszag, "Picosecond microwave pulses generated with a subpicosecond laser-driven semiconductor switch," Appl. Phys. Lett., vol. 39, no. 4, pp. 295-296, 1981.

[6] D. H. Auston, K. P. Cheung, and P. R. Smith, "Picosecond photoconducting Hertzian dipoles," Appl. Phys. Lett., vol. 45, no. 3, pp. 284-286, 1984.

[7] P. Smith, D. H. Auston, and M. C. Nuss, "Subpicosecond photoconducting dipole antennas," IEEE J. Quantum Electron., vol. 24, no. 2, pp. 255-260, Feb. 1988

[8] C. Fattinger and D. Grischkowsky, "Terahertz beams," Appl. Phys. Lett., vol. 54, no. 6, pp. 490-492, 1989.

[9] J. V. Rudd, D. A. Zimdars, and M. W. Warmuth, "Compact fiber-pigtailed terahertz imaging system," Proc. SPIE Commercial and Biomedical Applications of Ultrafast Lasers II, vol. 3934, pp. 27-35, 2000.

[10] J. V. Rudd, M. W. Warmuth, S. L. Williamson, and D. A. Zimdars, "Compact fiber pigtailed terahertz modules," U.S. Patent 6816 647, 2004.

[11] B. B. Hu, X.-C. Zhang, D. H. Auston, and P. R. Smith, "Free-space radiation from electro-optic crystals," Appl. Phys. Lett. vol. 56, no. 6, pp. 506-508, 1990.

[12] Q. Wu and X.-C. Zhang, "Free-space electro-optic sampling of terahertz beams," Appl. Phys. Lett., vol. 67, no. 24, pp. 3523-3525, 1995.

[13] M. Li, X.-C. Zhang, G. D. Sucha, and D. J. Harter, "Portable terahertz system and its applications," Proc. SPIE Commercial and Biomedical Applications of Ultrafast Lasers, vol. 3616, pp. 126-135, 1999.

[14] D. D. Arnone and C. M. Ciesla, "Method and apparatus for terahertz imaging," U.S. Patent 6957 099, 2005.

[15] M. Usami, M. Yamashita, K. Fukushima, C. Otani, and K. Kawase, "Terahertz wideband spectroscopic imaging based on two-dimensional electro-optic sampling technique," Appl. Phys. Lett. vol. 86, no. 14, art. no. 141109, 2005.

[16] B. Ferguson, S. Wang, D. Abbott, and X.-C. Zhang, "Powder retection with THz imaging," Proc. SPIE Terahertz for Military and Security Applications, vol. 5070, pp. 7-16, 2003.

[17] P. Coward and R. Appleby, "Development of an illumination chamber for indoor millimeter-wave imaging," Proc. SPIE Passive Millimeter-Wave Imaging Technology VI and Radar Sensor Technology VII, vol. 5077. pp. 54-61, 2003.
[18] C. Zandonella, "T-ray specs," Nature, vol. 424, pp. 721-722, 2003

[19] S. Hadjiloucas, L. S. Karatzas, and J. W. Bowen, "Measurements of leaf water content using terahertz radiation," IEEE Trans. Microw. Theory Tech., vol. 47, no. 2, pp. 142-149, Feb. 1999.

[20] P. Y. Han, G. C. Cho, and X.-C. Zhang, "Time-domain transillumination of biological tissues with terahertz pulses," Opt. Lett., vol. 25, no. 4, pp. 242-244, 2000.

[21] E. Berry, "Risk perception and safety issues," J. Biol. Phys., vol. 29, no. 2-3, pp. 263-267, 2003.

[22] M. Walther, B. M. Fischer, and P. U. Jepsen, "Noncovalent intermolecular forces in polycrystalline and amorphous saccharides in the far infrared," Chem. Phys., vol. 288, pp. 261-268, 2003

[23] L. B. Braly, K. Liu, M. G. Brown, F. N. Keutsch, R. S. Fellers, and R. J. Saykally, "Terahertz laser spectroscopy of the water dimer intermolecular vibrations. II. $\left(\mathrm{H}_{2} \mathrm{O}\right)_{2}$," J. Chem. Phys., vol. 112, no. 23 pp. $10314-10326,2000$

[24] B. M. Fischer, M. Walther, and P. U. Jepsen, "Far-infrared vibrational modes of DNA components studied by terahertz time-domain spectroscopy,' Phys. Med. Biol., vol. 47, pp. 3807-3814, 2002

[25] H. Harde, R. Cheville, and D. Grischkowsky, "Terahertz studies of collision-broadened rotational lines," J. Phys. Chem. A, vol. 101, no. 20, pp. 3646-3660, 1997.

[26] D. M. Mittleman, R. H. Jacobsen, R. Neelamani, R. G. Baraniuk, and M. C. Nuss, "Gas sensing using terahertz time-domain spectroscopy," Appl. Phys. B: Lasers Opt., vol. 67, no. 3, pp. 379-390, 1998.

[27] P. F. Bernath, Spectra of Atoms and Molecules, 2nd ed. Oxford, U.K.: Oxford University Press, 2005.

[28] R. H. Jacobsen, D. M. Mittleman, and M. C. Nuss, "Chemical recognition of gases and gas mixtures with terahertz waves," Opt. Lett., vol. 21, no. 24, pp. 2011-2013, 1996.

[29] B. M. Fischer, M. Hoffmann, H. Helm, G. Modjesch, and P. U. Jepsen, "Chemical recognition in terahertz time-domain spectroscopy and imaging," Semicond. Sci. Technol., vol. 20, pp. S246-S253, 2005

[30] M. van Exter and D. Grischkowsky, "Optical and electronic properties of doped silicon from 0.1 to $2 \mathrm{THz}$," Appl. Phys. Lett., vol. 56, no. 17 , pp. 1694-1696, 1990.

[31] T.-I. Jeon and D. Grischkowsky, "Nature of conduction in doped silicon," Phys. Rev. Lett., vol. 78, no. 6, pp. 1106-1109, 1997.

[32] I. Kudman, "A nondestructive measurement of carrier concentration in heavily doped semiconducting materials and its application to thin surface layers," J. Appl. Phys., vol. 34 no. 6, pp. 1826-1827, 1963.

[33] J. Ng and D. Abbott, "Introduction to solid-state quantum computation for engineers," Microelectron. J., vol. 33 no. 1-2, pp. 171-177, 2002.

[34] B. E. Cole, J. B. Williams, B. T. King, M. S. Sherwin, and C. R. Stanley, "Coherent manipulation of semiconductor quantum bits with terahertz radiation," Nature, vol. 410, pp. 60-63, 2001.
[35] H. S. Brandi, A. Latgé, and L. E. Oliveira, "Rabi oscillations, coherent properties, and model qubits in two-level donor systems under terahertz radiation," Phys. Rev. B, vol. 68, no. 23, art. no. 233206, 2003.

[36] B. Ferguson and X.-C. Zhang, "Materials for terahertz science and technology," Nature Materials, vol. 1, pp. 26-33, 2002.

[37] M. C. Beard, G. M. Turner, and C. A. Schmuttenmaer, "Terahertz spectroscopy," J. Phys. Chem. B, vol. 106 , no. 29 , pp. $7146-7159$, 2002.

[38] W. Withayachumnankul, B. Ferguson, T. Rainsford, S. P. Mickan, and D. Abbott, "Simple material parameter estimation via terahertz time-domain spectroscopy," IEE Electron. Lett., vol. 41, no. 14 pp. 800-801, 2005.

[39] T. Dorney, R. Baraniuk, and D. Mittleman, "Material parameter estimation with terahertz time-domain spectroscopy," J. Opt. Soc. Amer. A, vol. 18, no. 7 pp. 1562-1571, 2001.

[40] W. Withayachumnankul, H. Lin, S. P. Mickan, B. M. Fischer, and D. Abbott, "Analysis of measurement uncertainty in THz-TDS," Proc. SPIE Photonic Materials, Devices, and Applications II, vol. 6593, art. no. 659326, 2007.

[41] M. W. Schmidt, K. K. Baldridge, J. A. Boatz, S. T. Elbert, M. S. Gordon, J. H. Jensen, S. Koseki, N. Matsunaga, K. A. Nguyen, S. Su, T. L. Windus, M. Dupuis, and J. A. Montgomery, Jr., "General atomic and molecular electronic structure system," J. Comput. Chem., vol. 14, no. 11, pp. 1347-1363, 1993.

[42] M. J. Frisch et al., Gaussian 03, Revision B 0.5. Wallingford, CT: Gaussian Inc., 2004.

[43] F. L. Gervasio, G. Cardini, P. R. Salvi, and V. Schettino, "Low-frequency vibrations of all-trans-retinal: Far-infrared and Raman spectra and density functional calculations," J. Phys. Chem. A, vol. 102 no. 12, pp. 2131-2136, 1998.

[44] M. Walther, B. Fischer, M. Schall, H. Helm and P. U. Jepsen, "Far-infared vibrational spectra of all-trans retinal, 9-cis and 13-cis retinal measured by $\mathrm{THz}$ time-domain spectroscopy," Chem. Phys. Lett., vol. 332, no. 3-4, pp. 389-395, 2000.

[45] S. W. Lin, M. Groesbeek, I. van der Hoef, P. Verdegem, J. Lugtenburg, and R. A. Mathies, "Vibrational assignment of torsional normal modes of rhodopsin: Probing excited-state isomerization dynamics along the reactive $C-11=C-12$ torsion coordinate, J. Phys. Chem. B, vol. 102, no. 15, pp. 2787-2806, 1998.

[46] F. Blomgren and S. Larsson, "Exploring the potential energy surface of retinal, a comparison of the performance of different methods," J. Comput. Chem. vol. 26, no. 7, pp. 738-742, 2005.

[47] C. Morari and D. Bogdan, "A study of the anharmonic effects on the vibrational spectra of a realistic retinal chromophore model," Spectrochim. Acta A: Mol. Biomol. Spectrosc., vol. 61, no. 8, pp. 1881-1886, 2005.

[48] U. F. Röhrig, L. Guidoni, A. Laio, I. Frank, and U. Rothlisberger, "A molecular spring for vision," J. Amer. Chem. Soc., vol. 126 no. 47, pp. 15 328-15 329, 2004.

[49] L. Guo, Y. Hu, Y. Zhang, C. Zhang, Y. Chen, and X.-C. Zhang, "Vibrational spectrum 
of $\gamma$-HNIW investigated using terahertz time-domain spectroscopy," Opt. Express, vol. 14, no. 8, pp. 3654-3659, 2006.

[50] L. Ning, S. Jingling, S. Jinhai, L. Laishun, X. Xiaoyu, L. Meihong, and J. Yan, "Study on the THz spectrum of methamphetamine," Opt. Express, vol. 13, no. 18, pp. 6750-6755, 2005.

[51] Y. Chen, H. Liu, Y. Deng, D. Schauki, M. J. Fitch, R. Osiander, C. Dodson, J. B. Spicer, M. Shur, and X.-C. Zhang, "THz spectroscopic investigation of 2,4-dinitrotoluene," Chem. Phys. Lett., vol. 400, no. 4-6, pp. 357-361, 2004.

[52] M. R. Kutteruf, C. M. Brown, L. K. Iwaki, M. B. Campbell, T. M. Korter, and E. J. Heilweil, "Terahertz spectroscopy of short-chain polypeptides," Chem. Phys. Lett., vol. 375, no. 3-4, pp. 337-343, 2003.

[53] G. M. Day, J. A. Zeitler, W. Jones, T. Rades, and P. F. Taday, "Understanding the influence of polymorphism on phonon spectra: Lattice dynamics calculations and terahertz spectroscopy of carbamazepine," J. Phys. Chem. B, vol. 110, no. 1, pp. 447-456, 2006.

[54] A. Jubert, M. L. Legarto, N. E. Massa, L. L. Tévez, and N. B. Okulik, "Vibrational and theoretical studies of non-steroidal anti-inflammatory drugs Ibuprofen [2-(4-isobutylphenyl)propionic acid]; Naproxen [6-methoxy-a-methyl2-naphthalene acetic acid] and Tolmetin acids [1-methyl-5-(4-methylbenzoyl)-1Hpyrrole-2-acetic acid]," J. Mol. Struct., vol. 783, no. 1-3, pp. 34-51, 2006.

[55] T. Lo, I. S. Gregory, C. Baker, P. F. Taday, W. R. Tribe, and M. C. Kemp, "The very far-infrared spectra of energetic materials and possible confusion materials using terahertz pulsed spectroscopy," Vib. Spectrosc., vol. 42, no. 2, pp. 243-248, 2006.

[56] F. Huang, B. Schulkin, H. Altan, J. F. Federici, D. Gary, R. Barat, D. Zimdars, M. Chen, and D. B. Tanner, "Terahertz study of 1,3,5-trinitro-s-triazine by time-domain and Fourier transform infrared spectroscopy," Appl. Phys. Lett., vol. 85, no. 23, pp. 5535-5537, 2004.

[57] T. M. Korter, R. Balu, M. B. Campbell, M. C. Beard, S. K. Gregurick, and E. J. Heilweil, "Terahertz spectroscopy of solid serine and cysteine," Chem. Phys. Lett., vol. 418, no. 1-3, pp. 65-70, 2006.

[58] I. Jones, T. J. Rainsford, B. Fischer, and D. Abbott, "Towards T-ray spectroscopy of retinal isomers: A review of methods and modelling," Vib. Spectrosc., vol. 41, no. 2, pp. 144-154, 2006.

[59] Q. Wang, R. W. Schoenlein, L. A. Peteanu, R. A. Mathies, and C. V. Shank, "Vibrationally coherent photochemistry in the femtosecond primary event of vision," Science, vol. 266, no. 5184, pp. 422-424, 1994.

[60] R. A. Mathies, "Photons, femtoseconds and dipolar interactions: A molecular picture of the primary events in vision," in Proc. Novartis Found. Symp. Rhodopsins and Phototransduction, 1999, vol. 224, pp. 70-89.

[61] H. Kandori, Y. Shichida, and T. Yoshizawa, "Photoisomerization in rhodopsin," Biochem. (Moscow), vol. 66, no. 11, pp. 1197-1209, 2001.

[62] T. D. Lamb and E. N. Pugh, Jr., "Dark adaptation and the retinoid cycle of vision," Prog. Retinal Eye Res., vol. 23, no. 3, pp. 307-380, 2004.

[63] G. Wolf, "The visual cycle of the cone photoreceptors of the retina," Nutrition Rev., vol. 62, no. 7, pp. 283-286, 2004.

[64] V. Kuksa, Y. Imanishi, M. Batten, K. Palczewski, and A. R. Moise, "Retinoid cycle in the vertebrate retina: Experimental approaches and mechanisms of isomerization," Vis. Res., vol. 43, no. 28, pp. 2959-2981, 2003.

[65] Z. Buletic, K. J. Soprano, and D. R. Soprano, "Retinoid targets for the treatment of cancer," Crit. Rev. Eukaryot. Gene Expr., vol. 16, no. 3, pp. 193-210, 2006.

[66] O. Sorg, C. Antille, G. Kaya, and J. H. Saurat, "Retinoids in cosmeceuticals," Dermatol. Therapy, vol. 19, no. 5, pp. 289-296, 2006.

[67] L. Baumann, "Skin ageing and its treatment," J. Pathol., vol. 211, no. 2, pp. 241-251, 2007.

[68] N. Mirza, M. Fishman, I. Fricke, M. Dunn, A. M. Neuger, T. J. Frost, R. M. Lush, S. Antonia, and D. I. Gabrilovich, "All-Trans-retinoic acid improves differentiation of myeloid cells and immune response in cancer patients," Cancer Res., vol. 66, no. 18, pp. 9299-9307, 2006.

[69] J. A. Wass, "Modeling molecules," Science, vol. 290, no. 5499, p. 2098, 2000.

[70] A. D. Becke, "Density functional exchange energy approximation with asymptotic behaviour," Phys. Rev., vol. A38, no. 6, pp. 3098-3100, 1998.

[71] C. Lee, W. Yang, and R. G. Parr, "Development of the Colle-Salvetti correlation energy formula into a functional of the electron density," Phys. Rev. B, vol. 37, no. 2, pp. 785-789, 1988.

[72] W. J. Hehre, L. Radom, P. V. Schleyer, and J. Pople, Ab Initio Molecular Orbital Theory. New York: Wiley, 1986.

[73] R. J. Meier, "Calculating the vibrational spectra of molecules: An introduction for experimentalists with contemporary examples," Vib. Spectrosc., vol. 43, no. 1, pp. 26-37, 2007.

[74] S. P. Mickan, J. Munch, X.-C. Zhang, and D. Abbott, "Increased sensitivity in T-ray liquid spectroscopy using a rapid sample modulation," Proc. SPIE Terahertz and Gigahertz Electronics and Photonics III, vol. 5354, pp. 71-85, 2004.

[75] L. Duvillaret, F. Garet, and J.-L. Coutaz, "A reliable method for extraction of material parameters in terahertz time-domain spectroscopy," IEEE J. Sel. Topics Quantum Electron., vol. 2, no. 3, pp. 739-746, Sep. 1996.

[76] B. L. Yu, F. Zeng, Q. Xing, and R. R. Alfano, "Probing dielectric relaxation properties of liquid $\mathrm{CS}_{2}$ with terahertz time-domain spectroscopy," Appl. Phys. Lett., vol. 82, no. 26, pp. 4633-4635, 2003.

[77] I. H. Libon, M. Hempel, S. Seitz, N. E. Hecker, J. Feldmann, A. Hayd, G. Zundel, D. Mittleman, and M. Koch, "THz spectroscopy of polar liquids," Proc. SPIE Terahertz Spectroscopy and Applications, vol. 3617, pp. 24-29, 1999.

[78] T. Ikeda, A. Matsushita, M. Tatsuno, Y. Minami, M. Yamaguchi, K. Yamamoto, M. Tani, and M. Hangyo, "Investigation of inflammable liquids by terahertz spectroscopy," Appl. Phys. Lett., vol. 87, no. 3, art. no. 034105, 2005.
[79] S. Schrödle, B. Fischer, H. Helm, and R. Buchner, "Picosecond dynamics and microheterogenity of water + dioxane mixtures," J. Phys. Chem. A, vol. 111, no. 11, pp. 2043-2046, 2007.

[80] C. Rønne, L. Thrane, P.-O. Åstrand, A. Wallqvist, K. V. Mikkelsen, and S. R. Keiding, "Investigation of the temperature dependence of dielectric relaxation in liquid water by $\mathrm{THz}$ reflection spectroscopy and molecular dynamics simulation," J. Chem. Phys., vol. 107, no. 14, pp. 5319-5331, 1997.

[81] S. P. Mickan, R. Shvartsman, J. Munch, X.-C. Zhang, and D. Abbott, "Low noise laser-based T-ray spectroscopy of liquids using double-modulated differential time-domain spectroscopy," J. Opt. B: Quantum Semiclassical Opt., vol. 6, no. 8, pp. S786-S795, 2004.

[82] S. P. Mickan, K.-S. Lee, T.-M. Lu, J. Munch, D. Abbott, and X.-C. Zhang, "Double modulated differential THz-TDS for thin film dielectric characterization," Microelectron. J., vol. 33, pp. 1033-1042, 2002.

[83] K.-S. Lee, T.-M. Lu, and X.-C. Zhang, "The measurement of the dielectric and optical properties of nano thin films by THz differential time-domain spectroscopy," Microelectron. J., vol. 34, no. 1, pp. 63-69, 2003.

[84] J. Balakrishnan, B. M. Fischer, S. P. Mickan, and D. Abbott, "Investigation on improving the noise performance of T-ray liquid spectroscopy via double-modulated differential time-domain spectroscopy," Proc. SPIE Biomedical Applications of Micro- and Nanoengineering III, vol. 6416, art. no. 64160V, 2006.

[85] J. Balakrishnan, B. M. Fischer, S. P. Mickan, and D. Abbott, "Novel T-ray liquid spectroscopy via double-modulated differential time-domain spectroscopy," in Conf. Dig. 2006 Joint 31st Int. Conf. Infrared and Millimeter Waves and 14th Int. Conf. Terahertz Electronics, p. 446.

[86] X. Song and B. Swanson, "Direct, ultrasensitive, and selective optical detection of protein toxins using multivalent interactions," Anal. Chem., vol. 71, no. 11, pp. 2097-2107, 1999.

[87] A. Menikh, R. MacColl, C. A. Mannella, and X.-C. Zhang, "Terahertz biosensing, technology: Frontiers and progress," ChemPhysChem, vol. 3, no. 8, pp. 655-658, 2002.

[88] M. Nagel, P. H. Bolívar, M. Brucherseifer, H. Kurz, A. Bosserhoff, and R. Büttner, "Integrated $\mathrm{THz}$ technology for label-free genetic diagnostics," Appl. Phys. Lett., vol. 80, pp. 154-156, 2002.

[89] M. Nagel, P. H. Bolívar, M. Brucherseifer, H. Kurz, A. Bosserhoff, and R. Büttner, "Integrated planar terahertz resonators for femtomolar sensitivity label-free detection of DNA hybridization," Appl. Opt., vol. 41, no. 10, pp. 2074-2078, 2002.

[90] S. Mickan, A. Menikh, H. Liu, C. Mannella, R. MacColl, D. Abbott, J. Munch, and X.-C. Zhang, "Label-free bioaffinity detection using terahertz technology," Phys. Med. Biol., vol. 47, pp. 3789-3795, 2002.

[91] H.-B. Liu, G. Plopper, S. Earley, Y. Chen, B. Ferguson, and X.-C. Zhang, "Sensing minute changes in biological cell monolayers with $\mathrm{THz}$ differential time-domain spectroscopy," Biosens. Bioelectron., vol. 22, pp. 1075-1080, 2007. 
[92] R. M. Woodward, V. P. Wallace, R. J. Pye, B. E. Cole, D. D. Arnone, E. H. Linfield, and M. Pepper, "Terahertz pulse imaging of ex-vivo Basal Cell Carcinoma," J. Invest. Dermatol., vol. 120, no. 1, pp. 72-78, 2003.

[93] E. Pickwell, B. E. Cole, A. J. Fitzgerald, M. Pepper, and V. P. Wallace, "In vivo study of human skin using pulsed terahertz radiation," Phys. Med. Biol., vol. 49, no. 9, pp. 1595-1607, 2004.

[94] E. Berry, A. J. Fitzgerald, N. N. Zinov'ev, G. C. Walker, S. Homer-Vanniasinkam, C. D. Sudworth, R. E. Miles, J. M. Chamberlain, and M. A. Smith, "Optical properties of tissue measured using terahertz-pulsed imaging," Proc. SPIE Medical Imaging 2003: Physics of Medical Imaging, vol. 5030, pp. 459-470.

[95] G. M. Png, J.-W. Choi, I. Guest, B. W.-H. Ng, S. P. Mickan, D. Abbott, and X.-C. Zhang, "Molecular and structural preservation of dehydrated bio-tissue for THz spectroscopy," Proc. SPIE Biomedical Applications of Micro- and Nanoengineering III, vol. 6416, art. no. $64160 \mathrm{~W}, 2006$

[96] G. C. Walker, E. Berry, S. W. Smye, N. N. Zinov'ev, A. J. Fitzgerald, R. E. Miles, M. Chamberlain, and M. A. Smith, "Modelling the propagation of terahertz radiation through a tissue simulating phantom," Phys. Med. Biol., vol. 49, pp. 1853-1864, 2004.

[97] G. M. Png, S. P. Mickan, and D. Abbott, "The potential use of T-rays in diagnosing the head and brain," in Proc. Int. Workshop Terahertz Technology, M. Tonouchi, Ed., 2005, pp. 99-100.

[98] G. M. Png, S. P. Mickan, and D. Abbott, "Simulation of terahertz radiation in stratified media," Proc. SPIE Photonics: Design Technology, and Packaging II, vol. 6038, art. no. 60380M, 2005.

[99] C. Gabriel, S. Gabriel, and E. Corthout, "The dielectric properties of biological tissues: I. Literature survey," Phys. Med. Biol., vol. 41, no. 11, pp. 2231-2249, 1996.

[100] C. Gabriel and S. Gabriel, "Compilation of the dielectric properties of body tissues at RF and microwave frequencies, (accessed Oct. 14, 2005). [Online]. Available: http:// niremf.ifac.cnr.it/docs/DIELECTRIC/ Report.html

[101] J. Nolte, The Human Brain: An Introduction to Its Functional Anatomy, 5th ed. St. Louis, MO: Mosby, 2002

[102] J. Xu, K. W. Plaxco, and S. J. Allen, "Collective dynamics of lysozyme in water: Terahertz absorption spectroscopy and comparison with theory," J. Phys. Chem. B, vol. 110, pp. 24 255-24 259, 2006

[103] G. C. Walker, E. Berry, N. N. Zinov'ev, A. J. Fitzgerald, R. E. Miles, M. Chamberlain, and M. A. Smith, "Terahertz imaging and international safety guidelines," Proc. SPIE Medical Imaging 2002: Physics of Medical Imaging, vol. 4682, pp. 683-690, 2002.

[104] R. W. McGowan, G. Gallot, and D. Grischkowsky, "Propagation of ultrawideband short pulses of terahertz radiation through submillimeter-diameter circular waveguides," Opt. Lett., vol. 24 pp. 1431-1433, 1999.

[105] G. Gallot, S. P. Jamison, R. W. McGowan, and D. Grischkowsky, "Terahertz waveguides," J. Opt. Soc. Amer. B: Opt. Phys., vol. 17, no. 5, pp. 851-863, 2000

[106] S. P. Jamison, R. W. McGowan, and D. Grischkowsky, "Singlemode waveguide propagation and reshaping of sub-ps terahertz pulses in sapphire fibers," Appl.
Phys. Lett., vol. 76, no. 15, pp. 1987-1989, 2000

[107] R. Mendis and D. Grischkowsky, "Plastic ribbon $\mathrm{THz}$ waveguides," J. Appl. Phys., vol. 88, no. 7, pp. 4449-4451, 2000.

[108] R. Mendis and D. Grischkowsky, "Undistorted guided-wave propagation of subpicosecond terahertz pulses," Opt. Lett., vol. 26, no. 11, pp. 846-848, 2001.

[109] R. Mendis and D. Grischkowsky, "THz interconnect with low-loss and low-group velocity dispersion," IEEE Microw. Wireless Compon. Lett., vol. 11, no. 11, pp. 444-446, Nov. 2001.

[110] H. Han, H. Park, M. Cho, and J. Kim, "Terahertz pulse propagation in a plastic photonic crystal fiber," Appl. Phys. Lett., vol. 80, no. 15, pp. 2634-2636, 2002.

[111] T.-I. Jeon and D. Grischkowsky, "Direct optoelectronic generation and detection of sub-ps-electrical pulses on sub-mm-coaxial transmission lines," Appl. Phys. Lett., vol. 85, no. 25, pp. 6092-6094, 2004.

[112] K. Wang and D. M. Mittleman, "Metal wires for terahertz wave guiding," Nature, vol. 432, no. 7015, pp. 376-379, 2004.

[113] T.-I. Jeon, J. Zhang, and D. Grischkowsky, "THz Sommerfeld wave propagation on a single metal wire," Appl. Phys. Lett., vol. 86, art. no. 161904, 2005.

[114] A. Bingham, Y. Zhao, and D. Grischkowsky, "THz parallel plate photonic waveguide," Appl. Phys. Lett., vol. 87, art. no. 051101, 2005.

[115] T.-I. Jeon and D. Grischkowsky, "THz Zenneck surface wave ( $\mathrm{THz}$ surface plasmon) propagation on a metal sheet," Appl. Phys. Lett., vol. 88, art. no. 061113, 2006.

[116] R. Mendis, "Nature of subpicosecond terahertz pulse propagation in practical dielectric-filled parallel-plate waveguides," Opt. Lett., vol. 31, no. 17, pp. 2643-2645, 2006.

[117] M. Nagel, A. Marchewka, and H. Kurz, "Low-index discontinuity terahetz waveguides," Opt. Express, vol. 14, no. 21, pp. 9944-9954, 2006.

[118] S. Coleman and D. Grischkowsky, "A THz transverse electromagnetic mode two-dimensional interconnect layer cooperating quasi-optics," Appl. Phys. Lett. vol. 83, no. 18, pp. 3656-3658, 2003.

[119] L.-J. Chen, H.-W. Chen, T.-F. Kao, J.-Y. Lu, and C.-K. Sun, "Low-loss subwavelength plastic fiber for terahertz waveguiding," Opt. Lett., vol. 31, no. 3, pp. 308-310, 2006.

[120] L. Tong, R. R. Gattass, J. B. Ashcom, S. He, J. Lou, M. Shen, I. Maxwell, and E. Mazur, "Subwavelength-diameter silica wires for low-loss optical wave guiding," Nature, vol. 426, no. 6968, pp. 816-819, 2003.

[121] D. Mittleman, Sensing With Terahertz Radiation. Berlin, Germany: Springer, 2003

[122] S. Atakaramians, S. Afshar Vahid, H. Ebendorff-Heidepriem, B. M. Fischer T. Monro, and D. Abbott, "Terahertz waveguides and materials," in Conf. Dig. 2006 Joint 31st Int. Conf. Infrared and Millimeter Waves and 14th Int. Conf. Terahertz Electronics, 2006, p. 281.

[123] S. Atakaramians, S. Afshar Vahid, B. Fischer, H. Ebendorff-Heidepriem, T. Monro, and D. Abbott, "Microwire fibers for low-loss THz transmission," Proc. SPIE Smart Structures, Devices, and Systems III, vol. 6414, art. no. 64140I, 2006.
[124] A. W. Snyder and J. D. Love, Optical Waveguide Theory. London, U.K.: Chapman and Hall, 1995.

[125] K. Okamoto, Fundamentals of Optical Waveguides. San Diego, CA: Academic, 2000.

[126] X.-C. Long and S. R. J. Brueck, "Composition dependence of the photoinduced refractive-index change in lead silicate glasses," Opt. Lett., vol. 24 no. 16, pp. 1136-1138, 1999.

[127] N. Sugimoto, H. Kanbara, S. Fujiwara, K. Tanaka, Y. Shimizugawa, and K. Hirao, "Third-order optical nonlinearities and their ultrafast response in $\mathrm{Bi}_{2} \mathrm{O}_{3}-\mathrm{B}_{2} \mathrm{O}_{3}-\mathrm{SiO}_{2}$ glasses," J. Opt. Soc. Amer. B, vol. 16, no. 11, pp. 1904-1908, 1999.

[128] W. M. Elsasser, "Attenuation in a dielectric circular rod," J. Appl. Phys., vol. 20, no. 12, pp. 1193-1196, 1949.

[129] M. van Exter, C. Fattinger, and D. Grischkowsky, "Terahertz time-domain spectroscopy of water vapor," Opt. Lett. vol. 14, no. 20, pp. 1128-1130, 1989.

[130] H.-B. Liu, H. Zhong, N. Karpowicz, Y. Chen, and X.-C. Zhang, "Terahertz spectroscopy and imaging for defense and security applications," Proc. IEEE, vol. 95, no. 8 , Aug. 2007.

[131] A. J. Vander, J. H. Sherman, and D. S. Luciano, Human Physiology: The Mechanisms of Body Function. McGraw-Hill, 1990.

[132] K. Ryan and C. Ray, Sherris Medical Microbiology: An Introduction to Infectious Diseases. New York: McGraw-Hill, 2004.

[133] T. Yuan, J. Z. Xu, and X.-C. Zhang, "Development of terahertz wave microscopes," Infrared Phys. Technol., vol. 45, no. 5-6, pp. 417-425, 2004.

[134] S. Hunsche, M. Koch, I. Brener, and M. C. Nuss, "THz near-field imaging," Opt. Communications, vol. 150, no. 1 , pp. 22-26, 1998.

[135] O. Mitrofanov, I. Brener, R. Harel, J. D. Wynn, L. N. Pfeiffer, K. W. West, and J. Federici, "Terahertz near-field microscopy based on a collection mode detector," Appl. Phys. Lett., vol. 77, no. 22, pp. 3496-3498, 2000

[136] O. Mitrofanov, M. Lee, J. W. P. Hsu, I. Brener, R. Harel, J. F. Federici, J. D. Wynn, L. N. Pfeiffer, and K. W. West, "Collection-mode near-field imaging with 0.5-THz pulses," IEEE J. Sel. Topics Quantum Electron., vol. 7, no. 4, pp. 600-607, Jul./Aug. 2001.

[137] O. Mitrofanov, M. Lee, J. W. P. Hsu, L. N. Pfeiffer, K. W. West, J. D. Wynn, and J. F. Federici, "Terahertz pulse propagation through small apertures," Appl. Phys. Lett., vol. 79, no. 7, pp. 907-909, 2001.

[138] D. V. Palanker, G. M. H. Knippels, T. I. Smith, and H. A. Schwettman, "IR microscopy with a transient photo-induced near-field probe (tipless near-field microscopy)," Opt. Commun. vol. 148 , no. 4-6, pp. 215-220, 1998.

[139] Q. Chen and X.-C. Zhang, "Semiconductor dynamic aperture for near-field terahertz wave imaging," IEEE J. Sel. Topics Quantum Electron., vol. 7, no. 4, pp. 608-614, Jul./Aug. 2001.

[140] N. Klein, P. Lahl, U. Poppe, F. Kadlec, and P. Kuzel, "A metal-dielectric antenna for terahertz near-field imaging," J. Appl. Phys., vol. 98, art. no. 014910, 2005.

[141] M. Berta, S. Danylyuk, F. Kadlec, P. Kuzel, and N. Klein, "THz near-field spectroscopy 
based on metal dielectric antennae," in Conf. Dig. 2006 Joint 31st Int. Conf. Infrared and Millimeter Waves and 14th Int. Conf. Terahertz Electronics, 2006, p. 373.

[142] B. Knoll and F. Keilmann, "Near-field probing of vibrational absorption for chemical microscopy," Nature, vol. 399 no. 6732 , pp. $134-137,1999$.

[143] R. Hillenbrand, T. Taubner, and F. Keilmann, "Phonon-enhanced light-matter interaction at the nanometre scale," Nature, vol. 418, no. 6894, pp. 159-162, 2002.

[144] F. Zenhausern, Y. Martin, and H. Wickramasinghe, "Scanning interferometric apertureless microscopy: Optical imaging at 10 angstrom resolution," Science, vol. 269, no. 5227, pp. 1083-1085, 1995.

[145] N. C. J. van der Valk and P. C. M. Planken, "Electro-optic detection of subwavelength terahertz spot sizes in the near field of a metal tip," Appl. Phys. Lett., vol. 81, no. 9, pp. 1558-1560, 2002.

[146] A. Adam, J. Brok, A. van de Nes, and P. Planken, "Terahertz near-field measurements of field enhancement near metal objects," in Conf. Dig. 2006 Joint 31st Int. Conf. Infrared and Millimeter Waves and 14th Int. Conf. Terahertz Electronics, 2006, p. 15.

[147] K. Wang, A. Barkan, and D. Mittleman, "Sub-wavelength resolution using apertureless terahertz near-field microscopy," in Proc. Conf. Lasers and Electro-Optics (CLEO), 2003, p. 2.

[148] H.-T. Chen, R. Kersting, and G. C. Cho, "Terahertz imaging with nanometer resolution," Appl. Phys. Lett., vol. 83, no. 15, pp. 3009-3011, 2003.

[149] H. Park, J. Kim, M. Kim, and H. Hanl, "Terahertz near-field microscope," in Conf. Dig. 2006 Joint 31st Int. Conf. Infrared and Millimeter Waves and 14th Int. Conf. Terahertz Electronics, p. 13.

[150] T. Yuan, H. Park, J. Xu, H. Han, and X.-C. Zhang, "Field-induced THz wave emission with nanometer resolution," Proc. SPIE Smart Structures, Devices, and Systems II, vol. 5649, pp. 1-8, 2005.

[151] K. Wang, A. Barkan, and D. M. Mittleman, "Propagation effects in apertureless near-field optical antennas," Appl. Phys. Lett., vol. 84, no. 2, pp. 305-307, 2004.

[152] K. Wang, D. M. Mittleman, N. C. J. van der Valk, and P. C. M. Planken, "Antenna effects in terahertz apertureless near-field optical microscopy," Appl. Phys. Lett., vol. 85, no. 14, pp. 2715-2717, 2004.

[153] T. Yuan, S. Mickan, J. Xu, D. Abbott, and X.-C. Zhang, "Towards an apertureless electro-optic T-ray microscope," in Proc. Conf. Lasers and Electro-Optics (CLEO), 2002, pp. 637-638.

[154] T. Kiwa, M. Tonouchi, M. Yamashita, and K. Kawase, "Laser terahertz-emission microscope for inspecting electrical faults in integrated circuits," Opt. Lett., vol. 28, no. 21, pp. 2058-2060, 2003.

[155] M. Yamashita, T. Kiwa, M. Tonouchi, K. Nikawa, C. Otani, and K. Kawase, "Laser terahertz emission microscope for inspecting electrical failures in integrated circuits," in Proc. Int. Meeting for Future of Electron Devices, 2004, pp. 29-30.
[156] K. Wynne and D. Jaroszynski, "Superluminal terahertz pulses," Opt. Lett., vol. 24, no. 1, pp. 25-27, 1999.

[157] R. Lecaque, S. Grésillon, N. Barbey, R. Peretti, J.-C. Rivoal, and C. Boccara, "THz near-field optical imaging by a local source," Opt. Commun., vol. 262, no. 1, pp. 125-128, 2006.

[158] H. A. Bethe, "Theory of diffraction by small holes," Phys. Rev., vol. 66, no. 7-8, pp. 163-182, 1944.

[159] J. Z. Xu and X.-C. Zhang, "Optical rectification in an area with a diameter comparable to or smaller than the center wavelength of terahertz radiation," Opt. Lett., vol. 27, no. 12, pp. 1067-1069, 2002.

[160] G. L. Dakovski, B. Kubera, and J. Shan, "Localized terahertz generation via optical rectification in ZnTe," J. Opt. Soc. Amer. B Opt. Phys., vol. 22, no. 8, pp. 1667-1670, 2005.

[161] Q. Xing, L. Lang, Z. Tian, N. Zhang, S. Li, K. Wang, L. Chai, and Q. Wang, "The effect of two-photon absorption and optical excitation area on the generation of THz radiation," Opt. Commun., vol. 267, no. 2, pp. 422-426, 2006

[162] D. Abbott, B. Davis, B. Gonzalez, A. Hernandez, and K. Eshraghian, "Modelling of low power CW laser beam heating effects on a GaAs substrate," Solid-State Electron., vol. 42, no. 5, pp. 809-816, 1998.

[163] E. Hecht, Optics, 4th ed. San Francisco, CA: Addison-Wesley, 2002.

[164] A. Bennett, H. Jupnik, H. Osterberg, and O. Richards, Phase Microscopy: Principles and Applications. New York: Wiley, 1951.

[165] G. M. Png, S. P. Mickan, T. Rainsford, and D. Abbott, "Terahertz phase contrast imaging," Proc. SPIE Smart Structures, Devices, and Systems II, S. Al-Sarawi, Eds., vol. 5649, pp. 768-777, 2004.

[166] A. C. Kak and M. Slaney, Principles of Computerized Tomographic Imaging (Classics in Applied Mathematics). Philadelphia, PA: SIAM, 2001.

[167] D. M. Mittleman, S. Hunsche, L. Boivin, and M. C. Nuss, "T-ray tomography," Opt. Lett. vol. 22, no. 12, pp. 904-906, 1997.

[168] M. Fatemi and A. C. Kak, "Ultrasonic B-scan imaging: Theory of image formation and a technique for restoration," Ultrasonic Imaging, vol. 2, no. 1, pp. 1-47, 1980.

[169] H. Zhong, J. Xu, X. Xie, T. Yuan, R. Reightler, E. Madaras, and X.-C. Zhang, "Nondestructive defect identification with terahertz time-of-flight tomography," IEEE Sensors J., vol. 5, no. 2, pp. 203-208, Apr. 2005.

[170] A. J. Fitzgerald, B. E. Cole, and P. F. Taday, "Nondestructive analysis of tablet coating thicknesses using terahertz pulsed imaging," J. Pharm. Sci., vol. 94, no. 1, pp. 177-183, 2005.

[171] I. Geltner, D. Hashimshony, and A. Zigler, "Detection and electrical characterization of hidden layers using time-domain analysis of terahertz reflections," J. Appl. Phys., vol. 92, no. 1, pp. 203-206, 2002.

[172] J. Pearce, H. Choi, D. M. Mittleman, J. White, and D. Zimdars, "Terahertz wide aperture reflection tomography," Opt. Lett., vol. 30, no. 13, pp. 1653-1655, 2005.

[173] H. Stark, J. Woods, I. Paul, and R. Hingorani, "Direct Fourier reconstruction in computer tomography," IEEE Trans. Acoust., Speech, Signal Process., vol. ASSP-29, no. 2, pp. 237-245, Apr. 1981.

[174] A. C. Kak, "Computerized tomography with X-ray, emission, and ultrasound sources," Proc. IEEE, vol. 67, no. 9, pp. 1245-1272, Sep. 1979.

[175] R. Gordon, R. Bender, and G. T. Herman, "Algebraic Reconstruction Techniques (ART) for three-dimensional electron microscopy and X-ray photography," J. Theor. Biol., vol. 29, no. 3, pp. 471-481, 1970.

[176] G. T. Herman, Image Reconstruction From Projections - The Fundamentals of Computerized Tomography. New York: Academic, 1980.

[177] B. Ferguson, S. Wang, D. Gray, D. Abbott, and X.-C. Zhang, "Identification of biological tissue using chirped probe $\mathrm{THz}$ imaging," Microelectron. J., vol. 33, no. 12, pp. 1043-1051, 2002.

[178] S. Pan and A. Kak, "A computational study of reconstruction algorithms for diffraction tomography: Interpolation versus filtered-backpropagation," IEEE Trans. Acoust., Speech, Signal Process., vol. ASSP-31, no. 5, pp. 1262-1275, Oct. 1983.

[179] M. Slaney, A. C. Kak, and L. E. Larsen, "Limitations of imaging with first-order diffraction tomography," IEEE Trans. Microw. Theory Tech., vol. MTT-32, no. 8, pp. 860-874, Aug. 1984.

[180] A. J. Devaney, “A filtered backpropagation algorithm for diffraction tomography," Ultrasonic Imaging, vol. 4, no. 4, pp. 336-350, 1982.

[181] H. P. Baltes, Ed., Inverse Source Problem for Electromagnetic Prospecting. Berlin, Germany: Springer-Verlag, 1978.

[182] A. Ishimaru, Wave Propagation and Scattering in Random Media. New York: Academic, 1978.

[183] R. K. Mueller, M. Kaveh, and R. D. Iverson, "A new approach to acoustic tomography using diffraction techniques," in Acoustic Holography, vol. 8, A. Metherell, Ed. New York: Plenum, 1978.

[184] Q. Wu, T. D. Hewitt, and X.-C. Zhang, "Two-dimensional electro-optic imaging of THz beams," Appl. Phys. Lett., vol. 69, no. 8, pp. 1026-1028, 1996.

[185] X.-C. Zhang, "Three-dimensional terahertz wave imaging," Philos. Trans. Roy Soc. London A, vol. 362, pp. 283-299, 2004.

[186] X. X. Yin, B. W.-H. Ng, B. Ferguson, S. P. Mickan, and D. Abbott, "Statistical model for the classification of the wavelet transforms of T-ray pulses," in Proc. 18th Int. Conf. Pattern Recognition (ICPR), 2006, vol. 3, pp. 236-239.

[187] X. X. Yin, B. W.-H. Ng, B. Ferguson, S. P. Mickan, and D. Abbott, "2-D wavelet segmentation in 3-D T-ray tomography," IEEE Sensors J., vol. 7, no. 3, pp. 342-343, Mar. 2007.

[188] F. Rashid-Farrokhi, K. Liu, C. Berenstein, and D. Walnut, "Wavelet-based multiresolution local tomography," IEEE Trans. Image Process., vol. 6, no. 10, pp. 1412-1430, Oct. 1997.

[189] S. Wang, B. Ferguson, D. Abbott, and X.-C. Zhang, "T-ray imaging and tomography," J. Biological Phys., vol. 29, no. 2-3, pp. 247-256, 2003.

[190] C. C. Te, B. Ferguson, and D. Abbott, "Investigation of biomaterial classification using T-rays," Proc. SPIE Biomedical 
Applications of Micro- and Nanoengineering, vol. 4937, pp. 294-306, 2002.

[191] Y. C. Shen, T. Lo, P. F. Taday, B. E. Cole, W. R. Tribe, and M. C. Kemp, "Detection and identification of explosives using terahertz pulsed spectroscopic imaging," Appl. Phys. Lett., vol. 86, art. no. 241116, 2005.

[192] J. F. Federici, B. Schulkin, F. Huang, D. Gary, R. Barat, F. Oliveira, and D. Zimdars, "THz imaging and sensing for security applications-explosives, weapons and drugs," Semicond. Sci. Technol., vol. 20, pp. S266-S280, 2005.

[193] K. Kawase, Y. Ogawa, Y. Watanabe, and H. Inoue, "Non-destructive terahertz imaging of illicit drugs using spectral fingerprints," Opt. Express, vol. 11, no. 20 pp. 2549-2554, 2003.

[194] B. Ung, J. Balakrishnan, B. Fischer, B. W.-H. Ng, and D. Abbott, "Terahertz detection of substances for security related purposes," Proc. SPIE Smart Structures, Devices, and Systems III, vol. 6414, art. no. 64141U, 2006.

[195] B. M. Fischer, "Broadband THz time-domain spectroscopy of biomolecules-A comprehensive study of the dielectric properties of biomaterials in the far-infrared," Ph.D. dissertation, Dept. Mol. Opt. Phys., Freiburg Mater. Res. Center, Universität Freiburg, Freiburg, Germany, 2005.

\section{ABOUT THE AUTHORS}

Withawat withayachumnankul was born in Bangkok, Thailand, on December 20, 1980. He received the B.S. (Honors) and M.S. degrees in electronics engineering from King Mongkut's Institute of Technology at Ladkrabang (KMITL), Thailand, in 2000 and 2002, respectively. $\mathrm{He}$ is currently working toward the Ph.D. degree in the Adelaide T-ray Group, School of Electrical and Electronic Engineering, University of Adelaide, Adelaide, Australia, under Derek Abbott, Bernd Fischer, and Samuel Mickan.

Working at the Biomedical Signal and Image Processing Laboratory at KMITL in 2000, as a Postgraduate Student, he performed multidisciplinary research involving digital signal processing, medical image reconstruction, and computer graphics. Here, he played a major role in developing core programming codes for medical software currently deployed in many leading Thai institutions and hospitals. After receiving his M.S. degree, he served at his alma mater as a Lecturer, a position that he currently retains. In this role, he conducted the computer programming and computer graphics courses in the Department of Information Engineering. In 2005, he was also a Visiting Scholar at the University of Adelaide, under Derek Abbott. His current research involves T-ray signal processing, T-ray components, and superluminal (group delay) electromagnetic propagation.

Mr. Withayachumnankul was granted an Australian Endeavour International Postgraduate Research Scholarship (EIPRS) to study toward his Ph.D. degree in 2006.

Gretel M. Png (Student Member, IEEE) received the B.E. degree in electrical and electronics engineering from the University of Edinburgh, U.K., in 1997 and the M.S. degree in electrical engineering and computer science from the University of California, Irvine, in 2004. She is currently working toward the Ph.D. degree under Brian Ng, Derek Abbott, and Sam Mickan at the Centre for Biomedical Engineering, University of Adelaide, Adelaide, Australia.

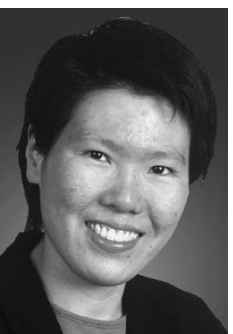

She worked as a systems engineer from 1997 to 2001 in Sweden and Israel. In 2006, she was a visiting scholar at Rensselaer Polytechnic Institute (RPI) under Ingrid Wilke and also under Xi-Cheng Zhang. Her research is in T-ray imaging and modeling.

Ms. Png won the Harold Dickinson Memorial Prize at the University of Edinburgh, the University of California at Irvine Excellence in Teaching Award, the prestigious Australian Postgraduate Award (APA) scholarship, the SPIE Educational Scholarship in Optical Science and Engineering (2006, 2007), and several University of Adelaide administered awards (Research Abroad Scholarship 2005, Mutual Community Postgraduate Travel Grant 2005, AUGU/RC Heddle Award 2006). She is actively involved in undergraduate teaching and the IEEE Student Branch at the University of Adelaide.
Xiaoxia (Sunny) Yin (Student Member, IEEE) was born in Dalian, China. She received the B.Eng. degree in industrial electronics from Dalian University, China. She is currently working toward the Ph.D. degree on three-dimensional terahertz computed tomography under Derek Abbott and Brian Ng, University of Adelaide, Adelaide, Australia.

She is also a Visiting Scholar at the University of Reading, U.K., under Silas Hadjiloucas and at the

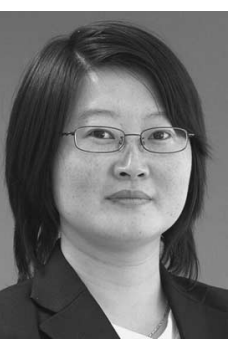
University of Cambridge, U.K., under Lynn F. Gladden. Her research interests include multiresolution analysis, segmentation, image reconstruction and classification, and their applications to T-ray imaging.

Ms. Yin received a series of awards, including the Ph.D. Scholarship of The University of Adelaide (2005), WavE Travel Fellowship from Switzerland (2006), The International Association for Pattern Recognition (IAPR) travel stipend from Hong Kong (2006), Research Abroad Scholarship from The University of Adelaide (2006), the Roger Pysden Memorial Fellowship from Australia Business, ABL State Chamber (2006), a student scholarship from 2007 IEEE International Symposium on Industrial Electronics in Vigo, Spain (2007), a Mutual Community Postgraduate Travel Grant from the University of Adelaide (2007), and an Overseas Travel Fellowship from The Australian Research Council Nanotechnology Network (ARCNN) (2007).

Shaghik Atakaramians was born in Tehran, Iran, on March 21, 1978. She received the B.S. degree from Iran University of Science and Technology (IUST) in 2000 and the M.S. degree from University of Tehran, Iran, in 2002. Her master's thesis was entitled "An equivalent network model for fullwave analysis of two-dimensional photonic crystals." She is currently working toward the Ph.D. degree in T-ray waveguides at the University of Adelaide, Adelaide, Australia, under Derek Abbott,

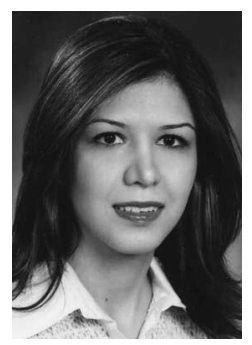
Bernd Fischer, Shahraam Afshar Vahid, and Tanya Monro.

In 2006, she was with the University of Adelaide as a Visiting Scholar under Derek Abbott.

Ms. Atakaramians was awarded the first prize for the best telecommunication graduate of the Department of Electrical Engineering, IUST, in 2000 , and first prize for the best telecommunication undergraduate in 1999 and the best job trainee of the electrical engineering faculty of IUST in the summer of 1999. She has also won a CLEO Milton Chang travel award administered by the Optical Society of America (OSA) in 2007. She was awarded an Endeavour International Postgraduate Research Scholarship in 2007 
Inke Jones was born in Bottrop, Germany, on August 15, 1977. She received the B.S. degree in medical laboratory science from the School for Medical Laboratory Scientists, Germany, in 2000 and the B.S. degree in biomedical engineering from the University of Applied Sciences, Germany, in 2004.

She joined the T-ray group at the University of Adelaide, Adelaide, Australia, as a visiting scholar in 2004 and is currently working on biomedical

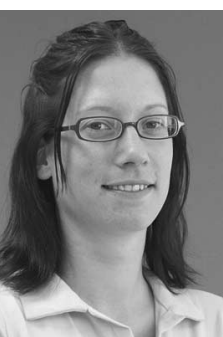
applications of terahertz spectroscopy and the prediction of vibrational modes in the terahertz region, under Bernd Fischer and Derek Abbott.
Hungyen Lin was born in Kaohsiung, Taiwan, R.O.C., on June 28,1982 . He received the B.Eng. degree (Honors) in computer systems engineering from the University of Adelaide, Adelaide, Australia, in 2003 and the M.S. degree from the University of South Australia (UniSA) on a Schefenacker Vision Systems funded scholarship on data mining and industrial applications of artificial neural networks in 2005. He is currently working toward the Ph.D. degree in electrical

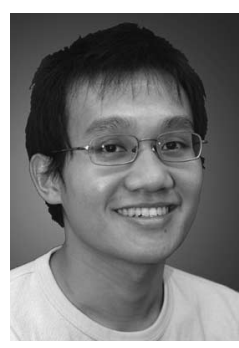
and electronic engineering at the University of Adelaide under Derek Abbott, Bernd Fisher, and Samuel Mickan on an Australian Postgraduate Award (APA) scholarship.

His current research involves T-ray near-field imaging and signal processing.
Brian W.-H. Ng (Member, IEEE) was born in Midlevel, Hong Kong, on May 12, 1974. He received the B.S. degree in mathematics and computer science in 1996 and the B.Eng. (Honors) and Ph.D. degrees in electrical and electronic engineering from the University of Adelaide, Adelaide, Australia, in 1997 and 2003, respectively.

Since 2002, he has been a Lecturer at the University of Adelaide in the School of Electrical and Electronic Engineering. His research interests

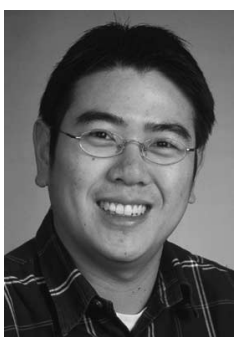
include signal processing, bioinspired engineering, distributed sensor networks, software-defined radio, and signal processing for T-ray imaging.

Dr. Ng was awarded the University of Adelaide medal for the top graduate in Electrical and Electronic Engineering during his studies. $\mathrm{He}$ is currently active in the South Australian chapter of the IEEE.
Benjamin Seam Yu Ung was born on August 30, 1982, in Adelaide, Australia. He received the B.Eng. degree (Honors) in computer systems engineering and the B.S. degree in mathematics and computer science from the University of Adelaide, in 2005. $\mathrm{He}$ is currently pursuing the Ph.D. degree in electrical engineering at The University of Adelaide under Derek Abbott, Bernd Fischer, and Brian Ng.

In 2007, he was also a Visiting Scholar at the Polytechnic University of Hong Kong collaborating under Charles Surya. His current research involves security applications of T-rays and novel T-ray photoconductive antennas.

Mr. Ung won a University of Adelaide Divisional Scholarship in 2006.

Jegathisvaran Balakrishnan was born on March 20, 1982, in Johor, Malaysia. He received the B.Eng. degree from University of Adelaide, Adelaide, Australia, in 2005. He is currently working toward the Ph.D. degree with the T-ray group of the University of Adelaide under Derek Abbott, Bernd Fischer, and Samuel Mickan.

$\mathrm{His}$ research interests are in T-ray liquid spectroscopy and security.

Mr. Balakrishnan was awarded the Post-

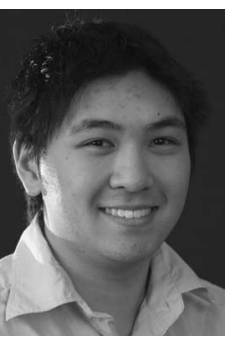

Bradley Ferguson (Member, IEEE) was born August 8, 1975, in Freemantle, Western Australia, $\mathrm{He}$ received the B.Eng. degree in electrical and electronic engineering and the Ph.D. degree (with Special Commendation) in the area of T-ray image processing under Derek Abbott and Douglas A Gray from the University of Adelaide, Adelaide, Australia, in 1997 and 2005, respectively.

He worked for Vision Abell Pty Ltd. for three years. In 2001 he was awarded a Fulbright Scholarship, and spent two years collaborating at X.-C. Zhang's group at Rensselaer Polytechnic Institute, Troy, NY. He is currently an Adjunct Senior Lecturer at the University of Adelaide and is concurrently employed by the Electronic Warfare Division of Tenix Systems Pty Ltd., Australia. His current research interests include THz-TDS and RF photonics.

Dr. Ferguson has won a number of honors and awards, including South Australian Employers' Chamber Prize in Electronic Engineering (1996), Institution of Radio and Electronics Engineer Prize in Microelectronics (1997), Philips Industries Holdings Ltd. Prize in Electronics (1997), South Australian Premier's Scholarship (1999), Australian Postgraduate Award (APA) scholarship (1999), Mutual Community Postgraduate Scholarship (2000), D. R. Stranks Postgraduate Scholarship (2000), Brenda Nettle Graduate Student Award (2003), New Focus Graduate Student Award (2003), University Alumni Medal (2005), The Gertrude Rohan Prize in Electronics (2006), and a Tall Poppy Award for science (2006). He was the President of the University of Adelaide student chapter of the IEEE (2000-2001) and is the South Australian State Secretary of Fulbright Alumni Association (2004-present). He is a member of the SPIE, LEOS, and OSA. was awarded the Sarawak Alumni Scholarship while working toward his B.S. degree. graduate Divisional Scholarship in 2006 for his research studies. H 
Samuel P. Mickan (Member, IEEE) was born on March 26, 1976, in Adelaide, Australia. He received the B.Eng. degree in electrical and electronic engineering, the B.A. degree in German and history, and the Ph.D. degree (with Special Commendation) under Derek Abbott and Jesper Munch, in the area of T-ray biosensing, at the University of Adelaide in 1997, 2002), and 2004, respectively.

Under a Fulbright Scholarship, he collaborated

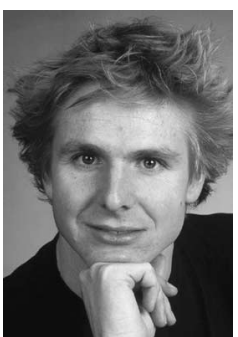
with X.-C. Zhang's group, Center for Terahertz Technology, Rensselaer Polytechnic Institute, Troy, NY, from 2001 to 2002. In 2003, he began lecturing in the School of Electrical and Electronic Engineering, University of Adelaide. He is currently both an Adjunct Senior Lecturer at the University of Adelaide and a Patent Attorney at Davies Collison Cave, Melbourne, Australia.

Dr. Mickan has won a number of awards and honors, including the Schneider Award for Excellence (1997), AFuW Barbara Crase Bursary (2001), George Murray Scholarship (2002), the Australian Academy of Sciences (AAS) Young Researcher's Award (2004), the University Medal from the University of Adelaide (2004), and the Tall Poppy Award for science (2005). He was Treasurer/Secretary of the Microwave Theory and Techniques Chapter of LEOS and was founder of the IEEE student branch at the University of Adelaide. Dr. Mickan is a member of the IEEE LEOS society, OSA, APS, and SPIE.

Bernd M. Fischer was born in Waldkirch, Germany. He received the Stage de Maitrise degree from the Université Paris-sud 11, France, in 1998 and the Diplom degree (with distinction) and the Ph.D. degree (summa cum laude) in physics, under Peter Uhd Jepsen and Hanspeter Helm, in the area of T-ray spectroscopy of biomolecules, from the University of Freiburg, Germany, in 2001 and 2006, respectively.

He joined the Adelaide T-ray Group, University

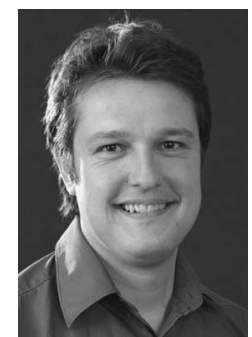
of Adelaide, Adelaide, Australia, in 2006.

Dr. Fischer was awarded the prestigious Research Council (ARC) Australian Postdoctoral (APD) Fellowship in 2007. He won an ERASMUS bursary in 1997-1998.
Derek Abbott (Fellow, IEEE) was born in London, U.K., on May 3, 1960. He received the B.Sc. degree (Honors) in physics from Loughborough University of Technology, U.K., in 1982 and the Ph.D. degree (with commendation) in electrical and electronic engineering, under Kamran Eshraghian and Bruce R. Davis, from the University of Adelaide, Adelaide, Australia, in 1995.

He has led a number of research programs in the imaging arena, ranging from the optical to

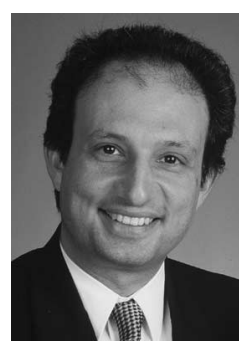
infrared to millimeter wave to T-ray (terahertz gap) regimes. From 1978 to 1986 , he worked at the GEC Hirst Research Centre, London, in the area of visible and infrared image sensors. His expertise also spans VLSI design, optoelectronics, device physics, and noise; where he has worked with nMOS, CMOS, SOS, CCD, GaAs, and vacuum microelectronic technologies. On migration to Australia, he worked for Austek Microsystems, Technology Park, South Australia, in 1986. Since 1987, he has been with the University of Adelaide, where he is presently a full Professor in the School of Electrical and Electronic Engineering and the Director of the Centre for Biomedical Engineering (CBME). He has appeared on national and international television and radio and has also received scientific reportage in New Scientist, The Sciences, Scientific American, Nature, The New York Times, and Sciences et Avenir. He holds over 300 publications/patents and has been an invited speaker at over 80 institutions, including Princeton, NJ; MIT, MA; Santa Fe Institute, NM; Los Alamos National Laboratories, NM; Cambridge, U.K.; and EPFL, Lausanne, Switzerland.

Prof. Abbott is a Fellow of the Institute of Physics (IOP), with honorary life membership. He won the GEC Bursary (1977), the Stephen Cole the Elder Prize (1998), the E. R. H. Tiekink Memorial Award (2002), SPIE Scholarship Award for Optical Engineering and Science (2003), the South Australian Tall Poppy Award for Science (2004) and the Premier's SA Great Award in Science and Technology for outstanding contributions to South Australia (2004). He has served as an editor and/or guest editor for a number of journals including IEEE JOURNAL OF SOLID-STATE CIRcuITS, Chaos (AIP), Smart Structures and Materials (IOP), Journal of Optics B (IOP), Microelectronics Journal (Elsevier), Fluctuation Noise Letters (World Scientific), and is currently on the Editorial Board of Proceedings of THE IEEE. He has served on a number of IEEE technical program committees, including the IEEE APCCS and the IEEE GaAs IC Symposium. 